\title{
Positronium in a Liquid Phase: Formation, Bubble State and Chemical Reactions
}

\author{
Sergey V. Stepanov, ${ }^{1}$ Vsevolod M. Byakov, ${ }^{1,2}$ Dmitrii S. Zvezhinskiy, ${ }^{1}$ Gilles Duplâtre, ${ }^{3}$ \\ Roman R. Nurmukhametov, ${ }^{1}$ and Petr S. Stepanov ${ }^{1}$
}

${ }^{1}$ Institute of Theoretical and Experimental Physics, Moscow 117218, Russia

${ }^{2}$ D. Mendeleev University of Chemical Technology of Russia, Miusskaya Square 9, Moscow 125047, Russia

${ }^{3}$ Institut Pluridisciplinaire Hubert Curien, CNRS/IN2P3, BP 28, 67037 Strasbourg Cedex 2, France

Correspondence should be addressed to Sergey V. Stepanov, stepanov@itep.ru

Received 2 April 2012; Revised 1 June 2012; Accepted 4 June 2012

Academic Editor: Michael Deleuze

Copyright (๑) 2012 Sergey V. Stepanov et al. This is an open access article distributed under the Creative Commons Attribution License, which permits unrestricted use, distribution, and reproduction in any medium, provided the original work is properly cited.

The present approach describes the $e^{+}$fate since its injection into a liquid until its annihilation. Several stages of the $e^{+}$evolution are discussed: (1) energy deposition and track structure of fast positrons: ionization slowing down, number of ion-electron pairs, typical sizes, thermalization, electrostatic interaction between $e^{+}$and the constituents of its blob, and effect of local heating; (2) positronium formation in condensed media: the Ore model, quasifree Ps state, intratrack mechanism of Ps formation; (3) fast intratrack diffusion-controlled reactions: Ps oxidation and ortho-paraconversion by radiolytic products, reaction rate constants, and interpretation of the PAL spectra in water at different temperatures; (4) Ps bubble models. Inner structure of positronium (wave function, energy contributions, relationship between the pick-off annihilation rate and the bubble radius).

\section{Introduction}

Positrons $\left(e^{+}\right)$as well as positronium atoms (Ps) are recognized as nanoscale probes of the local structure in a condensed phase (liquid or solid) and of the early radiolytic physicochemical processes occurring therein. The parameters of positron annihilation spectra determined experimentally (e.g., positron and Ps lifetimes, angular and energetic widths of the spectra, and Ps formation probability) are highly sensitive to the chemical composition, the local molecular environment of Ps (free volume size), and the presence of structural defects. They are also sensitive to variation of temperature, pressure, external electric and magnetic fields, and phase transitions.

The informative potentiality of positron spectroscopy strongly depends on the reliability of any theory describing the behavior of positrons in matter, since it should help decipher the information coded in the annihilation spectra. So, realistic models are needed for $e^{+}$track structure, $e^{+}$ energy losses, ionization slowing down and thermalization, intratrack reactions (ion-electron recombination, solvation, and interaction with scavengers), Ps formation process, Ps interaction with chemically active radiolytic species, and $e^{+} /$Ps trapping by structural defects.

Usually, treatment of the measured annihilation spectra is reduced to their resolution into a set of simple trial functions: sums of decaying time exponentials in the case of PALS (positron annihilation lifetime spectroscopy) and of Gaussians in the case of ACAR (angular correlation of annihilation radiation) and DBAR (doppler broadening of annihilation radiation). The outcome of such conventional analyses of positron annihilation data is the intensities of these components and the corresponding lifetimes/widths.

However, realistic theoretical models suggest more complex kinetics for describing physicochemical processes within the fast positron track, especially in its terminal part (which is called blob). Ps formation precisely occurs in the blob, and further Ps reactions with intratrack radiolytic products and solutes (oxidation, spin-conversion, and complex formation) also take place there. Because of inhomogeneous spatial distribution of the track species, their outdiffusion becomes an important factor: diffusion kinetics cannot be expressed 


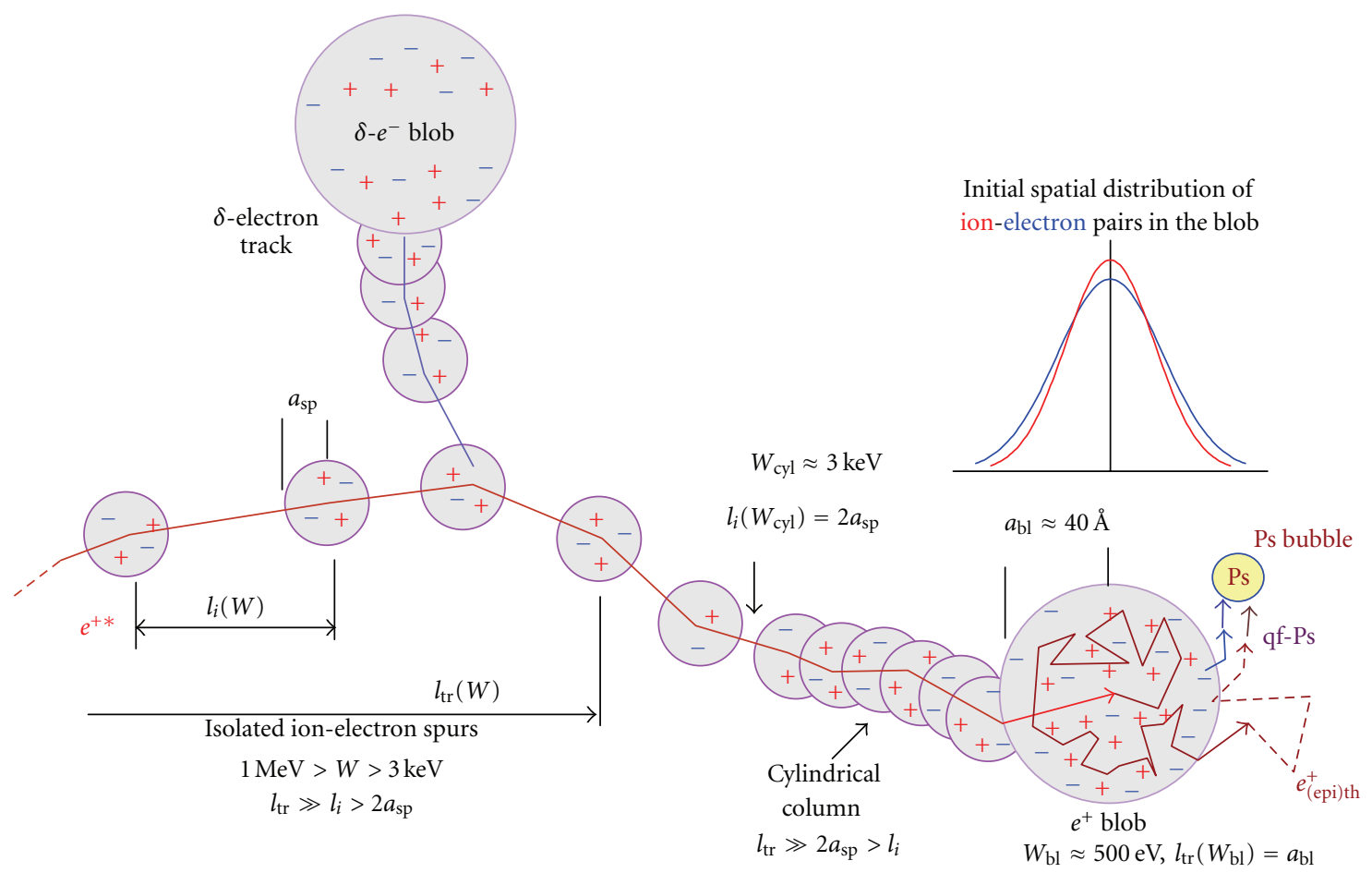

Figure 1: Scheme of the end part of the $e^{+*}$ track and Ps formation.

in terms of mere exponentials or Gaussians. Obviously, more elaborated theoretical models should be used in the fitting procedure of the annihilation spectra. In this case, the adjustable parameters (reaction rate constants or reaction radii, diffusion coefficients, initial size of the terminal part of the $e^{+}$track, and contact density in the Ps atom) would present a clear physical meaning instead of the abovementioned "intensities" of some trial functions.

Here we describe a model that can be used for interpretation of the positron spectroscopy data in molecular liquids. The model describes the $e^{+}$fate since its injection into a liquid until its annihilation.

\section{Energy Deposition and Track Structure of the Fast Positron}

2.1. Ionization Slowing Down. Positrons, produced in nuclear $\beta^{+}$-decay, have initial energies of about several hundreds of $\mathrm{keV}$. Once injected into a medium they lose energy via molecular ionization. Within 10 ps the positron energy drops down to the ionization threshold. Further approach to thermal equilibrium proceeds primarily via excitations of intra- and intermolecular vibrations. This usually takes few tens of picoseconds $[1,2]$.

Roughly half of the positron kinetic energy is lost in rare head-on collisions, resulting in the knocking out of $\delta$ electrons with kinetic energies of about several keV, the tracks of these electrons forming branches around the positron trail (Figure 1). The other half of the energy is spent in numerous glancing collisions with molecules. The average energy loss in such a collision is several tens of eV (up to $100 \mathrm{eV}$ ). A secondary electron knocked out in a glancing collision produces, in turn, a few ion-electron pairs inside a spherical nanovolume, called a "spur" in radiation chemistry. Its radius, $a_{\mathrm{sp}}$, is determined by the thermalization length of the knocked-out electrons in the presence of the Coulombic attraction of the parent ions. With a large uncertainty $a_{\text {sp }}$ in water may be estimated as 30-70 $\AA$ [3].

While the positron energy $W$ is greater than $W_{\text {cyl }} \sim$ $3 \mathrm{keV}$, the mean distance $l_{i}$ between adjacent ionizations produced by the positron is greater than the spur size $2 a_{\text {sp }}$. It ensues that at high positron energies, the spurs are well separated from each other. The trajectory of the fast positron is a quasistraight line because $l_{i}$ is less than the positron transport path $l_{\mathrm{tr}}$. The latter is the mean distance traveled by the positron before it changes its initial direction of motion by $90^{\circ}$.

When $l_{i}<2 a_{\mathrm{sp}}<l_{\mathrm{tr}}$ or $W_{\mathrm{bl}}<W<W_{\text {cyl }}$ the spurs overlap, forming something like a cylindrical ionization column. When the $e^{+}$energy becomes less than the blob formation energy, $W_{\mathrm{bl}}(<1 \mathrm{keV}$, see below), the positron is about to create a terminal blob. The diffusion motion of $e^{+}$in the blob becomes more pronounced: the direction of its momentum changes frequently due to elastic scattering and the ionization of surrounding molecules. All intrablob ionizations are confined within a sphere of radius $a_{\mathrm{bl}}$. The terminal positron blob contains a few tens of ion-electron pairs $\left(n_{0} \approx W_{\mathrm{bl}} / W_{\text {iep }} \approx 30\right)$ because the average energy $W_{\text {iep }}$ required to produce one ion-electron pair is $16-22 \mathrm{eV}$ [4]. Finally, the positron becomes subionizing and its energy loss rate drops by almost 2 orders of magnitude [5]. 
The dependences of $l_{i}(W)$ and $l_{\mathrm{tr}}(W)$ versus the $e^{+}$ energy in liquid water are shown in Figure 2 [6]. The calculation of the mean distance between adjacent ionizations $l_{i}(W)=W_{\text {iep }} / \operatorname{LET}(W)$ is based on $e^{-}$slowing down data (http://physics.nist.gov/PhysRefData/Star/Text/ ESTAR.html) and the estimation of the transport path has been done in the framework of the Born approximation (the Born amplitude was calculated by simulating an $\mathrm{H}_{2} \mathrm{O}$ molecule as an isoelectronic atom).

As we have mentioned, $e^{+}$moves in a diffusive way at the blob formation stage. So its spatial displacement squared should increase "linearly" with time, but because $e^{+}$diffusion coefficient, $D_{p}(W)$, is energy dependent, this relationship becomes more complicated. When $e^{+*}$ loses energy from $W_{i}$ down to $W_{f}$, its mean square displacement is [7]

$$
\begin{aligned}
R_{\mathrm{ion}}^{2}\left(W_{i}, W_{f}\right) & =\int_{t\left(W=W_{f}\right)}^{t\left(W=W_{i}\right)} 6 D_{p}(W) d t \\
& =\int_{W_{f}}^{W_{i}} l_{\mathrm{tr}}(W) \frac{d W}{|-d W / d x|_{\text {ion }}} .
\end{aligned}
$$

Here we used

$$
\begin{gathered}
D_{p}(W)=\frac{l_{\mathrm{tr}}(W) v_{p}}{6}, \\
d t=\frac{d x}{v_{p}}=\frac{1}{v_{p}} \cdot \frac{d W}{|-d W / d x|_{\text {ion }}}
\end{gathered}
$$

and $v_{p}$ is the positron velocity

The blob parameters, $W_{\mathrm{bl}}$ and $a_{\mathrm{bl}}$, can be estimated from the following equations, Figure 3:

$$
l_{\mathrm{tr}}\left(W_{\mathrm{bl}}\right)=a_{\mathrm{bl}}, \quad R_{\mathrm{ion}}^{2}\left(W_{\mathrm{bl}}, \mathrm{Ry}\right)-a_{\mathrm{bl}}^{2}=a_{\mathrm{bl}}^{2} .
$$

The first relationship implies that the positron with energy $W_{\mathrm{bl}}$ "jumps" to the center of the nascent blob from aside, passing the distance $l_{\mathrm{tr}}\left(W_{\mathrm{bl}}\right)$ (the red arrow in Figure 3 ). After that, the positron is located at the center of the blob. The second equation corresponds to the rest of the $e^{+}$diffusion motion (its slowing down till the subionizing energies; $\mathrm{Ry}=13.6 \mathrm{eV}$ stands here for a typical value of these energies). All this terminal part of the $e^{+}$trajectory must be confined within the positron blob (i.e., within the sphere with the radius $\left.a_{\mathrm{bl}}\right)$, that is, the $e^{+}$displacement squared, $R_{\mathrm{ion}}^{2}\left(W_{\mathrm{bl}}, \mathrm{Ry}\right)-a_{\mathrm{bl}}^{2}$, equals the dispersion of the ion-electron pairs of the blob, $a_{\mathrm{b} l}^{2}$.

In the case of liquid water one obtains $W_{\mathrm{bl}} \approx 500 \mathrm{eV}$ and $a_{\mathrm{bl}} \approx 40 \AA$. It seems that values of $a_{\mathrm{bl}}$ and $W_{\mathrm{bl}}$ should not differ significantly from one liquid to another because the ionization slowing down parameters depend mostly on the ionization potential and the average electron density, parameters that are more or less the same in all molecular media.

2.2. Thermalization Stage. Interaction between the Positron and Its Blob. At the end of the slowing down by ionization and electronic excitation, the spatial distribution of the positron coincides with the distribution of the blob species (i.e., $\left.\sim \exp \left(-r^{2} / a_{\mathrm{bl}}^{2}\right)\right)$. Such a subionizing

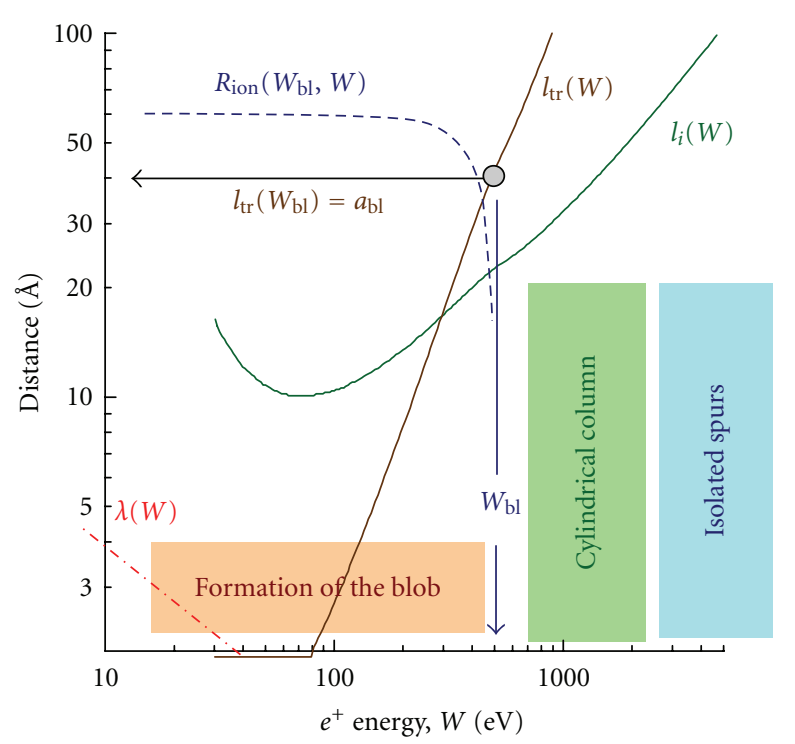

FIGURE 2: Dependences of $l_{i}(W)$ and $l_{\text {tr }}(W)$ versus the energy $W$ of the positron in liquid water. This figure illustrates also the solution of (3) for the blob parameters $W_{\mathrm{bl}}$ and $a_{\mathrm{bl}}: l_{\mathrm{tr}}\left(W_{\mathrm{bl}}\right)=$ $a_{\mathrm{bl}}, R_{\mathrm{ion}}\left(W_{\mathrm{bl}}, \mathrm{Ry}\right)=\sqrt{2 a_{\mathrm{bl}}^{2}}=\sqrt{2} a_{\mathrm{bl}}$. Red dash-and-dot line displays the de Broglie wavelength of the positron, $\lambda=h / m v \approx$ $12.3[\AA] / \sqrt{W, \mathrm{eV}}$.

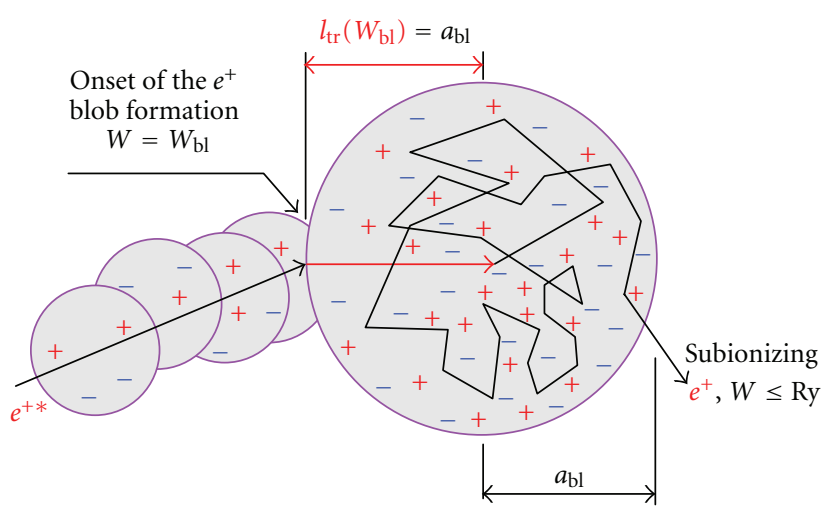

FIgURE 3: Scheme of the terminal positron blob.

positron having some $\mathrm{eV}$ of excess kinetic energy may easily escape from its blob. It is expected that by the end of thermalization, the $e^{+}$distribution becomes broader with the dispersion:

$$
a_{p}^{2} \approx a_{\mathrm{bl}}^{2}+\left\langle R_{\mathrm{vib}}^{2}\left(W_{0}, T\right)\right\rangle_{W_{0}} .
$$

Here, $R_{\text {vib }}^{2}\left(T, W_{0}\right)$ is determined by analogy with $R_{\text {ion }}^{2}$, see (1), where $|d W / d x|_{\text {ion }}$ should be replaced by $|d W / d x|_{\text {vib }}$, because the stopping power of subionizing positrons relates to the excitation of vibrations, but not to ionizations ( $T$ is the temperature in energy units). The estimation of $a_{p}$ requires quantitative data on $|d W / d x|_{\text {vib }}$, scattering properties of the subionizing positron, and the spectrum of its energies $W_{0}$ just after the last ionization event. In $(4)\langle\cdots\rangle_{W_{0}}$ denotes the average over $W_{0}$. In contrast to the parameters related to ionization slowing down, $a_{p}$ depends on the properties of the 
investigated liquid and may reach hundreds of $\AA$ especially if the liquid is nonpolar.

Between the positively charged ions and the knockedout intrablob electrons there exists a strong Coulombic attraction: outdiffusion of the electrons (even during their thermalization) is almost completely suppressed and their distribution is close enough to that of the ions. This case is known as ambipolar diffusion when ions and electrons expand with the same diffusion coefficient equal to the duplicated diffusion coefficient of the ions. The electrostatic potential in the blob comes out everywhere positive, that is, repulsive towards the positron (typical value of this repulsive energy is about several $T$ ) [7].

However, there is an opposite effect: while residing inside the blob, the thermalized $e^{+}$rearranges the intrablob electrons so that the total energy of the system decreases because of the Debye screening. The corresponding energy drop may be estimated by using the Debye-Huckel theory. It is $\sim e^{2} / \mathcal{E}\left(r_{D}+a_{\mathrm{bl}} / n_{0}^{1 / 3}\right)$ where $\varepsilon$ is the high frequency dielectric permittivity. However, the Debye radius $r_{D} \approx$ $\left(4 \pi r_{c} c_{\text {iep }}\right)^{-1 / 2} \approx 4 \AA$ is quite small in comparison with $a_{\mathrm{bl}} / n_{0}^{1 / 3}$ the average distance between intrablob electrons (here $r_{c}=$ $e^{2} / \varepsilon T$ is the Onsager radius and $c_{\text {iep }} \approx n_{0} /(4 / 3) \pi a_{\mathrm{bl}}^{3}$ is the concentration of ion-electron pairs within the blob). Thus, this screening energy $\sim T n_{0}^{1 / 3} r_{c} / a_{\mathrm{bl}}$ becomes a dominant contribution, which is some tenths of eV. It may result in a trapping of the positron, which may finally thermalize close to the central part of its blob. Sometimes this version of the blob model describing Ps formation is called the "black blob model" [8] in contrast to the "white blob model," where this effect of in-blob trapping of thermalized and also slightly epithermal positrons is neglected $[9,10]$.

\subsection{Effects of Local Heating and Premelting in the Terminal} Part of the $e^{+}$Track. We have estimated above that the ionization slowing down of energetic positrons $\left(e^{+*}\right)$ and subsequent ion-electron recombination release an energy up to $1 \mathrm{keV}$ in the terminal $e^{+}$blob, this energy being finally converted into heat. Therefore, the temperature in the $e^{+}$blob should be higher than the bulk temperature. This phenomenon may be called the local heating effect. This transient temperature regime may strongly affect, for example, the Ps bubble growth, by changing the viscosity of the medium. This effect may also influence the mobility of intratrack species and their reaction rates constants.

For quantitative estimations we simulate this process with the help of the macroscopic heat transfer equation [12]:

$$
c_{p} \rho \frac{\partial T(r, t)}{\partial t}=\operatorname{div}(\lambda \nabla T)+q_{+}(r, t), \quad T(r, t=0)=T_{\text {bulk }} .
$$

Here, $T(r, t)$ is the local temperature, $T_{\text {bulk }}$ is the bulk temperature of the medium, $c_{p}$ is its specific heat capacity, $\rho$ is the density, and $\lambda$ is the thermal conductivity. The second term in the RHS quantifies the energy released by the positron when creating its blob: $q_{+}(r, t) \approx W_{\mathrm{bl}} G(r, a) f(t, \tau)$, where $W_{\mathrm{bl}} \approx 1 \mathrm{keV}$ is the blob formation energy, $G(r, a) \approx$ $e^{-r^{2} / a_{\mathrm{bl}}^{2}} / \pi^{3 / 2} a_{\mathrm{bl}}^{3}$ describes the spatial distribution of the released energy, and $f(t, \tau) \approx \exp \left(-(t-1 \mathrm{ps})^{2} / 2 \tau^{2}\right) /(\sqrt{2 \pi} \tau)$ is its temporal distribution, where $\tau \approx 0.3$ ps is the typical time of ion-electron recombination.

In general, the system can be simultaneously solid and liquid. Then, the following method for obtaining a numerical solution of (5) can be used [12, 19]. To describe the deposition of the latent heat of melting, $q_{m}$, we added to $c_{p}(T)$ a term, which is nonzero only in a narrow temperature interval $T_{m}-\Delta T<T<T_{m}$, where $T_{m}$ is the melting temperature and $\Delta T$ is the width of the phase transition (arbitrarily fixed to $0.25 \mathrm{~K}$ ). Moreover, an integration of this term over temperature must give $q_{m}$. This additional overshot to $c_{p}(T)$ is simulated by a Gaussian function as follows:

$$
\tilde{c}_{p}(T)=c_{p}(T)+\frac{q_{m} \exp \left(-\left(T-T_{m}\right)^{2} / 2 \Delta T^{2}\right)}{\sqrt{2 \pi \Delta T^{2}}} .
$$

Close to the phase transition region, the $T$-dependences of thermal conductivity and density were approximated by smooth functions:

$$
\begin{aligned}
& \lambda(T)=\frac{\lambda^{S}}{\exp \left(-\left(T_{m}-T\right) / \Delta T\right)+1}+\frac{\lambda^{L}}{\exp \left(\left(T_{m}-T\right) / \Delta T\right)+1}, \\
& \rho(T)=\frac{\rho^{S}}{\exp \left(-\left(T_{m}-T\right) / \Delta T\right)+1}+\frac{\rho^{L}}{\exp \left(\left(T_{m}-T\right) / \Delta T\right)+1} .
\end{aligned}
$$

This approach works well especially when it is hard to trace an interphase boundary.

Simulations were done for water, methanol, ethanol, and butanol. Some thermodynamic properties of these substances are given in Table 1. Temperature profiles $T(r, t)$ were calculated numerically within a spherically symmetric volume for $r<200 \AA$ and for $t$ up to 1 ns. Equation (5) was solved with the boundary condition $T(r=$ $200 \AA)=T_{\text {bulk }}$ with the help of PDEPE subroutine from Matlab. Some temperature profiles in ice and in water close to the melting point are shown in Figure 4.

Close to the phase transition region, the state of the medium at a given spatial point may be deduced from its local temperature: if $T<T_{m}-\Delta T$, it is solid, and for $T>T_{m}+\Delta T$ we have a liquid; in between there is a "mixed" region. Figure 5 displays the maximum radius, $R_{\max }$, of the molten region versus $T_{\text {bulk }}-T_{m}$ and the lifetime $t_{\mathrm{ex}}$ of the molten region. At $t>t_{\mathrm{ex}}$ temperature of any point of the medium is below its melting point.

The local heating effect may result in a so-to-say "preliminary" formation of the so-called Ps bubble state. (It is a common stand point that in a liquid phase Ps atom forms a nanobubble and resides therein. Nature of this state is discussed below.) While the major fraction of the medium is solid, Ps bubble may be formed within the small premelted region close to the origin of the $e^{+}$blob. This effect can 
TABLE 1: Thermodynamical properties for water and some alcohols in solid and liquid phases near melting temperature [20-24].

\begin{tabular}{lcccc}
\hline Substance & $T_{m}, \mathrm{~K}$ & $\lambda^{S} / \lambda^{L}, \mathrm{~W} / \mathrm{m} / \mathrm{K}$ & $q_{m}, \mathrm{~J} / \mathrm{g}$ & $0.98 / 0.79$ \\
Methanol & 175.6 & $0.32 / 0.21$ & 99 & 109 \\
Ethanol & 159 & $0.27 / 0.17$ & 125 & $1.06 / 0.79$ \\
Butanol & 184.5 & $0.47 / 0.16$ & 334 & $1.05 / 0.81$ \\
Water & 273 & $2.38 / 0.56$ & $0.92 / 1.00$ \\
\hline
\end{tabular}
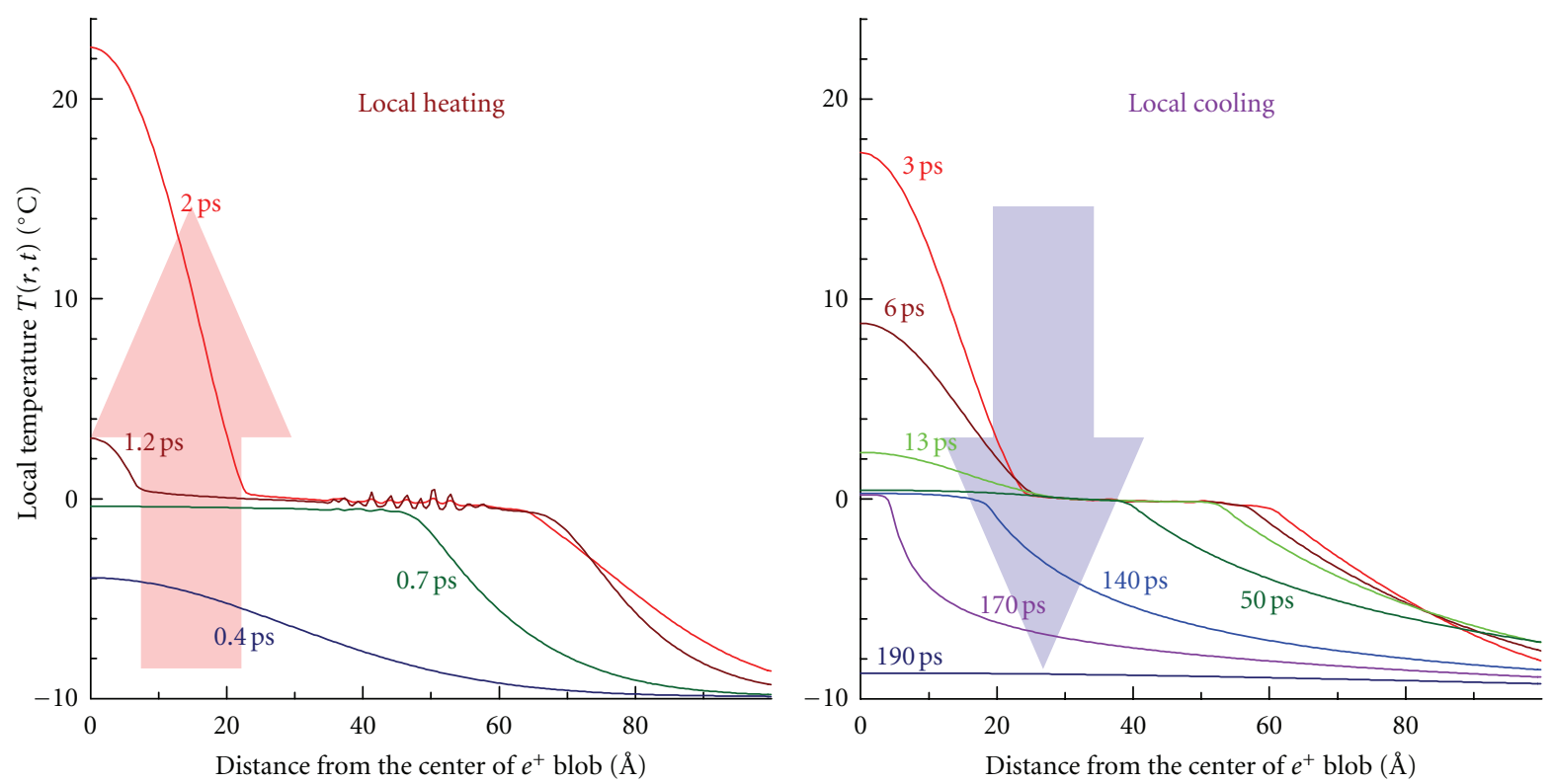

(a)
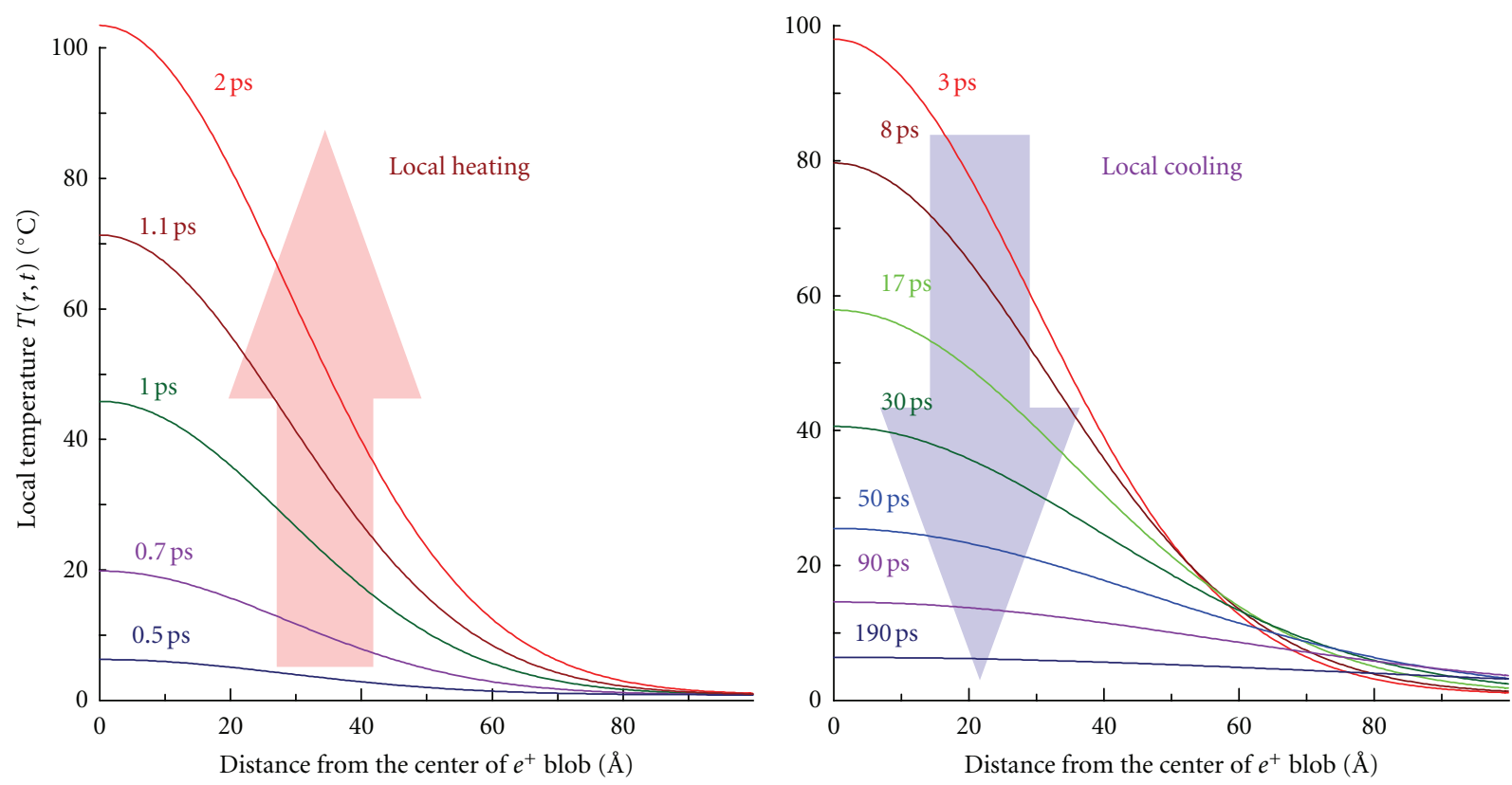

(b)

FIgURE 4: Calculated temperature profile in ice, $T_{\text {bulk }}=-10^{\circ} \mathrm{C}$ (on top), and in water, $T_{\text {bulk }}$ (bottom), is slightly above $0^{\circ} \mathrm{C}$. In both cases $a_{\mathrm{bl}}=40 \AA, W_{\mathrm{bl}}=1 \mathrm{keV}$. 


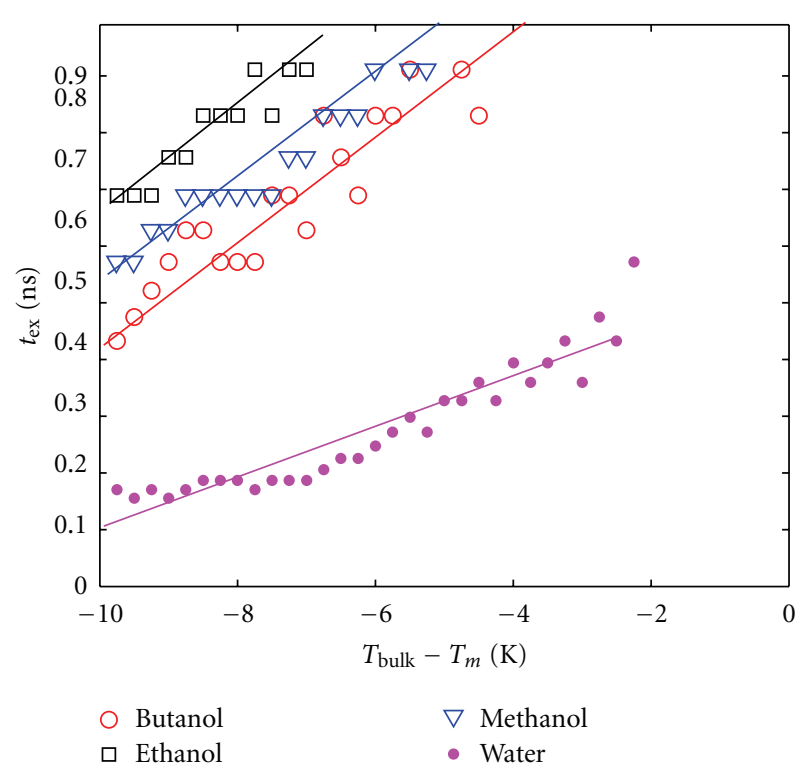

(a)

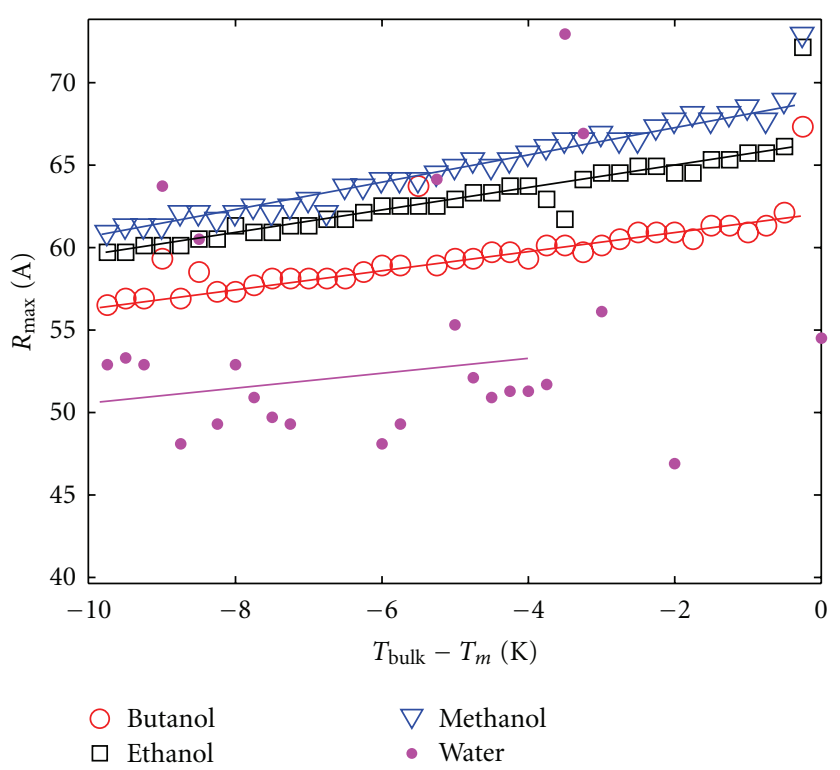

(b)

FIGURE 5: Lifetime of the molten region $t_{\mathrm{ex}}$ (a) and its radius $R_{\max }$ (b) for methanol, ethanol, butanol, and water are displayed as a function of the difference of the bulk temperature and the melting temperature.

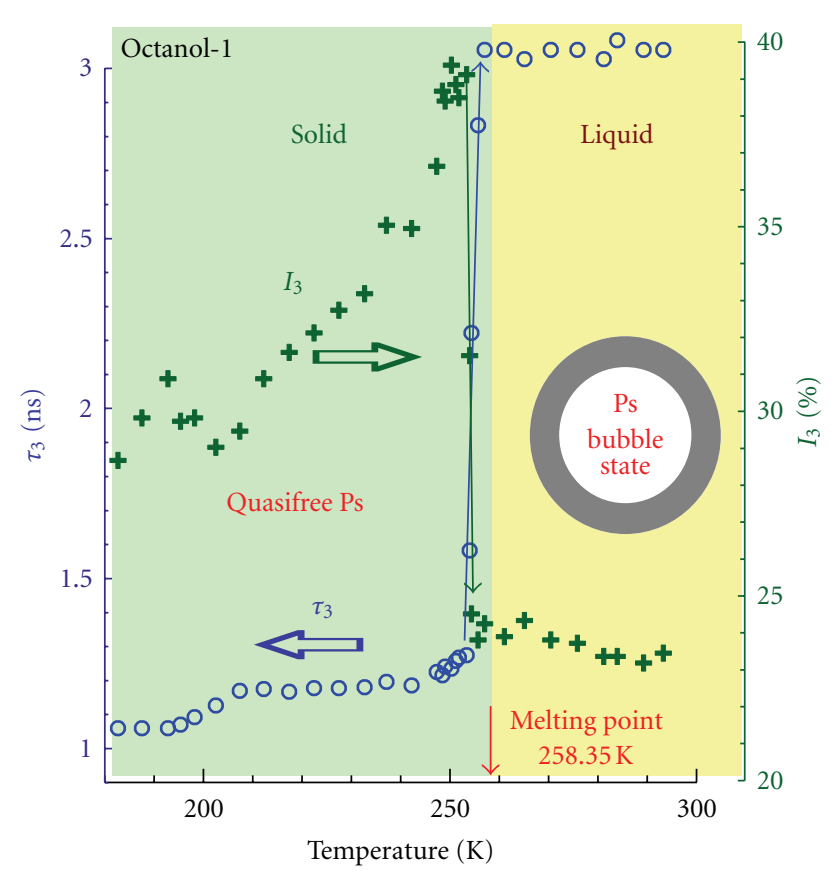

Figure 6: Temperature dependences of the ortho-Ps lifetime and the intensity of the corresponding lifetime component in octanol [11].

be observed experimentally as an increase of the long-lived (ortho-Ps) lifetime, $\tau_{3}$, from its solid phase value to the value corresponding to the liquid state. This behavior is illustrated in Figure 6. This transient temperature regime may also affect, for example, the Ps bubble growth (through the viscosity), the mobility of intratrack species, and their reaction rates coefficients.

\section{Positronium Formation in Condensed Media}

3.1. The Ore Model. The Ore model was proposed for interpretation of the Ps formation in gases [25]. It implies that the "hot" positron, $e^{+*}$, having excess kinetic energy, pulls out an electron from a molecule $M$, thereby forming a Ps atom and leaving behind a positively charged radicalcation $\mathrm{M}^{+\cdot}$ :

$$
e^{+*}+\mathrm{M} \longrightarrow \mathrm{Ps}+\mathrm{M}^{+\cdot}
$$

This process is most effective when the $e^{+*}$ energy $W$ lies within the "Ore gap":

$$
I_{G}-\frac{\mathrm{Ry}}{2}<W<W_{\mathrm{ex}} \quad\left(\text { or } I_{G}\right)
$$

Here $I_{G}$ is the first ionization potential of the molecule $\mathrm{M}$, $W_{\text {ex }}$ is its electronic excitation threshold, and Ry/2 $=6.8 \mathrm{eV}$ is the Ps binding energy in a gas phase. If the positron energy is lower than $I_{G}-\mathrm{Ry} / 2, e^{+}$cannot pick up an electron from a molecule. When $W>W_{\text {ex }}$, electronic excitations and ionizations dominate and Ps formation becomes less effective.

3.2. Quasifree Ps State. In the condensed phase, because of the presence of molecules and lack of free space, the final Ps state differs from that in vacuum [7]. We call this state quasifree positronium (qf-Ps). If we adopt a binding energy $E_{b}$ of qf-Ps in a dielectric continuum of roughly $\mathrm{Ry} / 2 \varepsilon^{2}$ (instead of just Ry/2 in vacuum), where $\varepsilon=n^{2} \approx 2$ high-frequency dielectric permittivity is the square of the 
refractive index, it is seen that the Ore gap in a medium gets squeezed

$$
I_{L}-\frac{\mathrm{Ry}}{2 \varepsilon^{2}}<W<W_{\mathrm{ex}}^{L}
$$

and may even completely disappear because of a significant decrease in the binding energy. Here, $W_{\mathrm{ex}}^{L}$ is the lower threshold of electronic excitations of a molecule in a liquid, $I_{L} \approx I_{G}-V_{0}^{-}-\left|U_{p}\right|$ is the liquid phase ionizing potential of a molecule, $V_{0}^{-}$is the energy of the ground state of an electron in a medium (sometimes it is called the $e^{-}$work function, Table 2), and $U_{p}$ is the energy of polarization interaction of the positively charged ion with the medium.

3.3. Mechanism of Ps Formation. The above discussion points out the unique possibility for the Ps formation mechanism in molecular media. It postulates that Ps is formed through the combination of the thermalized particles quasifree positron and one of the intratrack electrons:

$$
\mathrm{e}_{\mathrm{qf}}^{+}+e_{\mathrm{blob}}^{-} \longrightarrow\left(e^{+} \cdots e^{-}\right) \longrightarrow \mathrm{qf}-\mathrm{Ps} \longrightarrow \mathrm{Ps} \text { in the bubble. }
$$

This reaction proceeds in the terminal part of the $e^{+}$track (in the $e^{+}$blob). If the positron is thermalized outside the blob, the only way for it to form Ps is to diffuse back and pick up one of the intrablob electrons. Otherwise it annihilates as a "free" positron. When $e^{+}$picks up an electron, the "initial" separation between them is comparable to the average distance $\left(4 \pi a_{\mathrm{bl}}^{3} / 3 n_{0}\right)^{1 / 3} \approx 20 \AA$ between intrablob species. The binding energy of such a $e^{+} \cdots e^{-}$pair is small, about $0.1 \mathrm{eV}$. So, the translational kinetic energies of the particles must be less than the binding energy, otherwise the pair will break up. Thus, just before Ps formation, the positron and track electron must be almost thermalized (note that the total energy of this pair at this stage is approximately the sum $V_{0}^{+}+V_{0}^{-}$of the $e^{+}$and $e^{-}$work functions), Figure 7 .

However, such a weakly bound pair is not at the bottom of its energy spectrum. The two particles approach each other (in average) and continue to release energy via excitation of molecular vibrations. Finally, the pair reaches an intermediate equilibrium state, which we term the quasifree Ps. Roughly, the qf-Ps binding energy is $E_{b} \approx \mathrm{Ry} / 2 \varepsilon^{2}$, about $1 \mathrm{eV}$. In liquids, energy gain of the $e^{+} e^{-}$pair is related with the rearrangement of molecules and appearance of some additional free space around it. $e^{+}$and $e^{-}$may get closer, repelling molecules from their location and forming a Ps bubble state. A substantial decrease in their Coulombic energy is the driving force of this process.

Over the last 30 years this combination mechanism has become extremely widespread $[25,27]$. It has been used to interpret numerous data on Ps chemistry and explain variations of the Ps yields (from 0 to 0.7 ) in very different condensed media, where parameters of the Ore gap are practically the same. It provides a natural explanation to the changes in the Ps formation probability at phase transitions. Experimentally, the observed monotonic inhibition of Ps yields (practically down to zero) in solutions of electron acceptors contradicts the Ore model but inserts well in the
TABle 2: Values of $V_{0}^{-}$for different liquids at room temperature [26].

\begin{tabular}{lc}
\hline Liquid & $V_{0}^{-}, \mathrm{eV}$ \\
\hline Helium; 4.2 K & 1.3 \\
n-dodecane & 0.2 \\
n-decane & 0.18 \\
n-heptane & 0.12 \\
n-hexane & 0.1 \\
Nitrogen; 77.3 K & 0.05 \\
n-pentane, c-hexane & 0.01 \\
Argon; 86.4 K & 0 \\
Benzene & -0.14 \\
Isooctane & -0.17 \\
Toluene & -0.22 \\
Neopentane & -0.38 \\
MeOH, EtOH, PrOH & -0.4 \\
Xenon; 170 K & -0.57 \\
Water & -1.2 \\
\hline
\end{tabular}

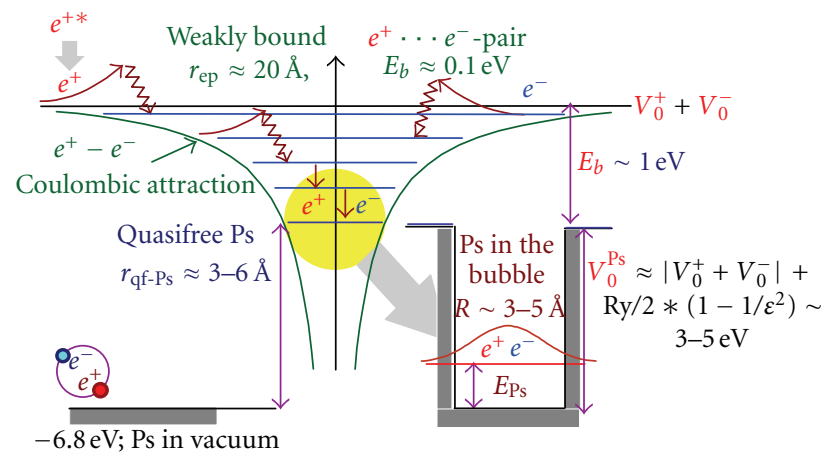

Figure 7: Mechanism of the Ps formation. Here, for simplicity, the potential in which Ps is localized is shown as a rectangular well.

recombination mechanism. It explains the anti-inhibition effect, including experiments on Ps formation in moderate electric fields in pure liquids and mixtures.

There are two models that utilize this mechanism, the spur model [28] and the blob model (or diffusionrecombination model) $[29,30]$. In spite of the fact that both models answer the question about the Ps precursor in the same way, they differ as to what constitutes the terminal part of the $e^{+}$track and how to calculate the probability of the Ps formation [7]. In the following we shall consider the blob model.

\section{Intratrack Reactions in the Positron Blob}

The interaction of the Ps atom with primary radiolytic products, formed in the terminal part of the positron track (in the $e^{+}$blob) due to $e^{+}$ionization slowing down, is a feature inherent to the positron spectroscopy of any molecular medium. Many intratrack products are strong oxidizers and/or radicals (like $\mathrm{H}_{3} \mathrm{O}^{+}$radical cations and $\mathrm{OH}$ radicals in water) and their initial concentration in the 


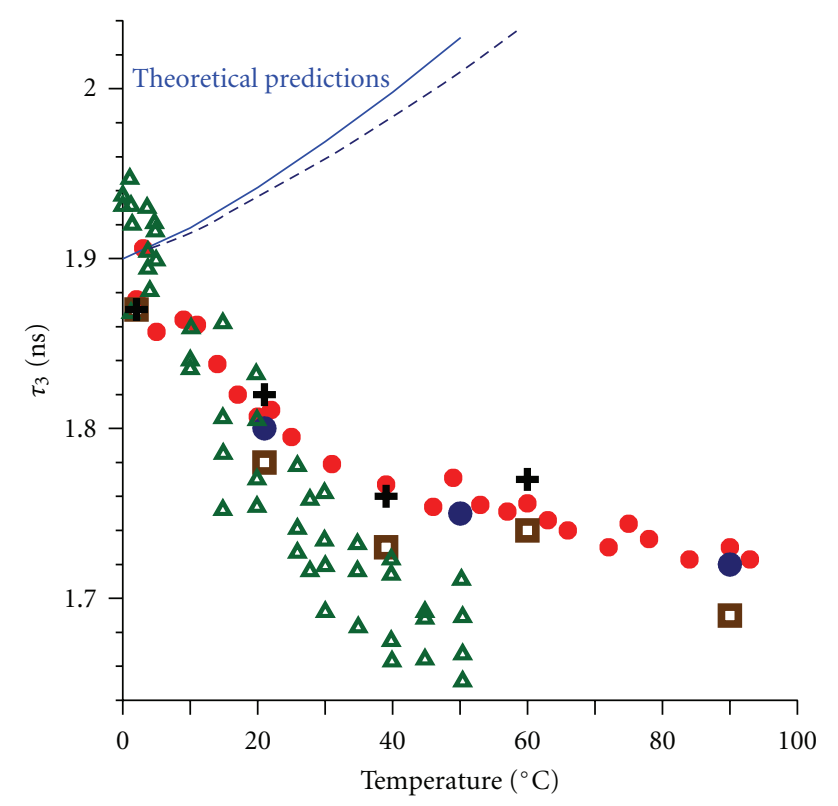

Pure $\mathrm{H}_{2} \mathrm{O}$
Stepanov et al., 2011
A Kaltera et al., 2005
ㅁ Duplâtre et al., 1985
A Abbé et al., 1984
Talamoni et al., 1982

Figure 8: Temperature dependence of the lifetime, $\tau_{3}$, of the longlived component in pure water. Experimental data are derived from a 3-exponential deconvolution of the spectra [13-17] and theoretical predictions are based on the standard models of the Ps bubble (finite potential well: solid line; infinite potential well: dashed line) [18].

$e^{+}$blob is not small (up to $0.05 \mathrm{M}$ ). The average distance between intrablob species is comparable with the diffusion displacement of the Ps bubble before $e^{+}$annihilation, so the contribution of diffusion-controlled oxidation and orthoparaconversion reactions is quite possible.

Neglecting these reactions leads to obvious contradictions. Figure 8 shows the $T$-dependence of the lifetime $\left(\tau_{3}\right)$ of the long-lived component of PAL spectra in pure water (roughly, $\tau_{3}$ is the pick-off lifetime of ortho positronium). Experimental data from different authors reasonably agree, but all are in strong contradiction with the theoretical expectations based on any Ps bubble model [18]: with increasing $T$, the surface tension coefficient decreases, so the size of the Ps bubble should increase, which should lead to an increase in $\tau_{3}$ with $T$, in sharp contradiction to the PAL data. So one of the most informative ways to investigate Ps intratrack reactions is to study the temperature variation of the PAL spectra.

In liquid water, the Ps bubble growth proceeds very fast ( $\lesssim 10$ ps) and so it may be considered as an instantaneous process [31]. Further intratrack radiolytic processes are known rather well there. In glycerol at temperatures $\lesssim 50^{\circ} \mathrm{C}$, formation of the Ps bubble state and further Ps interaction with intratrack radiolytic products are rather slow [32]. Of course, all these processes strongly depend on temperature,

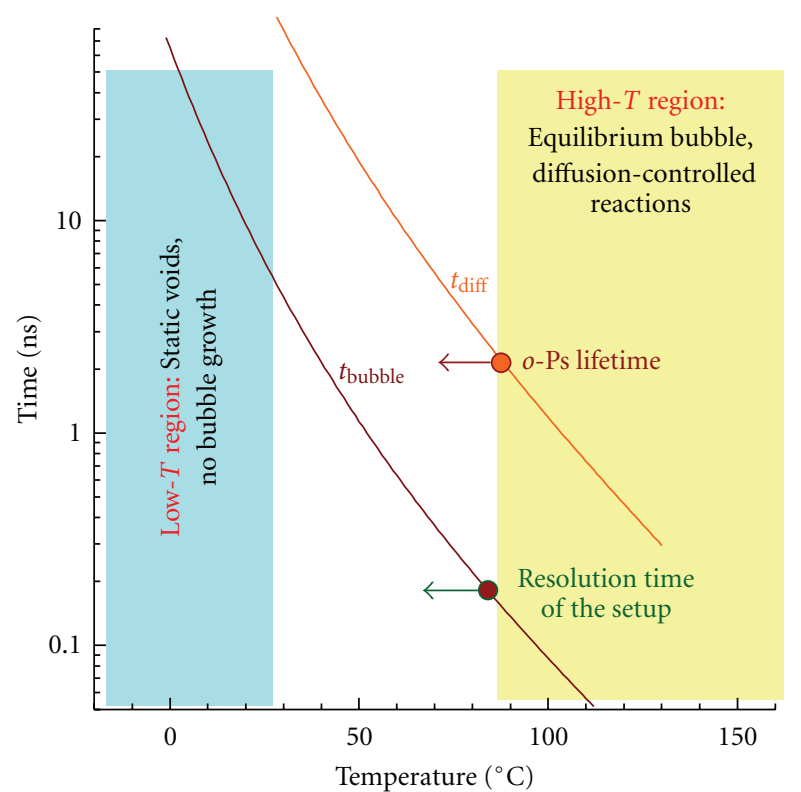

Figure 9: Temperature dependences of the Ps bubble formation time $t_{\text {bubble }}$ and typical time $t_{\text {diff }}$ of the diffusion-controlled intrablob reactions in glycerol.

so firstly it is worth to consider two extreme cases, the cases of "high" and "low" temperatures.

4.1. High-Temperature Region. This region may be defined through the following conditions:

(1) the Ps bubble formation time, $t_{\text {bubble }}(T) \approx R \eta / \sigma$ [31] is short as compared to the time resolution of a PAL spectrometer, $\sim 0.2 \mathrm{~ns}$. Here, $R \approx 3-6 \AA$ is the radius of the Ps bubble and $\eta$ and $\sigma$ are the viscosity and surface tension coefficients of a liquid medium. Experimental observation of the bubble growth is impossible in this case because the equilibrium state is reached too fast (less than $0.1 \mathrm{~ns}$ ).

(2) the diffusion length $\sqrt{6\left(D_{\mathrm{Ps}}+D_{i}\right) \tau_{3}}$ of Ps and radiolytic products (subscript $i$ ) in the $e^{+}$blob during the orthoPs lifetime, $\tau_{3}$ (about few nanoseconds), must exceed the average distance between intrablob particles $\bar{r} \approx 10-20 \AA$. This can be expressed through the condition $t_{\text {diff }}<\tau_{3}$, where $t_{\text {diff }}=\bar{r}^{2} / 6\left(D_{i}+D_{\text {Ps }}\right)$; on the basis of the StokesEinstein relationship, $D_{i} \approx D_{\mathrm{Ps}} \approx k_{B} T / 6 \pi \eta(T) R_{i}, R_{i}$ is a few $\AA$. In glycerol both conditions are satisfied at the same temperatures above $90^{\circ} \mathrm{C}$ (Figure 9), but in liquid water they are fulfilled at any temperature from 0 to $100^{\circ} \mathrm{C}$.

In the high- $T$ region the influence of intrablob reactions is important. The radiolytic processes initiated through ionizations induced by the slowing down of fast $e^{+*}$ may be represented through the following basic reactions:

ionization:

$$
e^{+*}+\mathrm{RH} \longrightarrow e^{-}+\dot{\mathrm{R}} \mathrm{H}^{+}+e^{+},
$$

ion-molecule reaction:

$$
\dot{\mathrm{R}} \mathrm{H}^{+}+\mathrm{RH} \longrightarrow \mathrm{RH}_{2}^{+}+\dot{\mathrm{R}}
$$


ion-electron recombination:

$$
\begin{gathered}
e^{-}+\dot{\mathrm{R}} \mathrm{H}^{+} \longrightarrow \cdot \dot{\mathrm{R}} \mathrm{H}_{\text {tripl }}^{*}, \mathrm{RH}_{\text {singl }}^{*}, \\
\mathrm{e}^{-}+\mathrm{RH}_{2}^{+} \longrightarrow \mathrm{RH}+\dot{\mathrm{H}} \longrightarrow \dot{\mathrm{R}}+\mathrm{H}_{2},
\end{gathered}
$$

electron solvation (in polar media; question about $e^{+}$ solvation remains open):

$$
\mathrm{e}^{-} \longrightarrow e_{s}^{-},
$$

electronic deexcitation:

$$
\cdot \dot{\mathrm{R}} \mathrm{H}_{\text {tripl }}^{*} \stackrel{+\mathrm{RH}}{\longrightarrow} 2 \dot{\mathrm{R}}+\mathrm{H}_{2}, \quad \mathrm{RH}_{\text {singl }}^{*} \longrightarrow \mathrm{RH} .
$$

From reactions $((12)-(15))$ one can infer that the total number of $\dot{\mathrm{R}}$ in the $e^{+}$blob varies weakly in time and is approximately equal to the initial number, $n_{0}$, of ionelectron pairs in the blob. However, their spatial distribution broadens in time due to outdiffusion.

As we have discussed above, Ps formation is described by reaction (11), which may be briefly written as follows:

$$
\mathrm{e}^{+}+e^{-} \longrightarrow \text { Ps. }
$$

The main effect of ions and radicals on Ps is oxidation and spin conversion reactions. If we denote for simplicity all these species $\left(\dot{\mathrm{R}} \mathrm{H}^{+}, \mathrm{RH}_{2}^{+}, \dot{\mathrm{R}}, \dot{\mathrm{H}}\right)$ by the same symbol, $\dot{\mathrm{R}}$, and term them "radicals," these species lead to

Ps oxidation:

$$
\mathrm{Ps}+\dot{\mathrm{R}} \longrightarrow e^{+}+\mathrm{R}^{-},
$$

ortho-paraconversion:

$$
\text { para- or ortho-Ps }+\dot{\mathrm{R}} \longrightarrow \frac{1}{4} \text { para-Ps }+\frac{3}{4} \text { ortho-Ps }+\dot{\mathrm{R}} \text {. }
$$

In the high-temperature region these Ps reactions are mostly diffusion controlled. With the help of the nonhomogeneous chemical kinetics approach (the "white" blob model) $[6$, 18] reactions (12)-(20) may be described in terms of the following equations

$$
\begin{gathered}
\frac{\partial c_{i}(r, t)}{\partial t}=D_{i} \Delta c_{i}-k_{i j} c_{i} c_{j}-\lambda_{i} c_{i}, \\
c_{i}(r, 0)=c_{i}^{0} \frac{\exp \left(-r^{2} / a_{i}^{2}\right)}{\pi^{3 / 2} a_{i}^{3}},
\end{gathered}
$$

where $c_{i}(r, t)$ is the concentration of the intrablob species of the ith type (including all the positron states; note that the initial spatial distribution of the $e^{+}$is usually somewhat broader than that of ion-electron pairs in the blob. This is due to the appearance of the local electric field which pushes $e^{+}$out of the blob and keeps track electrons close to the ions, see Section 2.2), $k_{i j}$ is the rate constant of the reaction between the $i$ and $j$ reactants and $\lambda_{i}$ is the decay rate of the $i$ th particles including possible annihilation.

According to the Smoluchowski approach, the reaction rate constant $k_{\mathrm{Ps}, \mathrm{R}}$ (let us take as an example reactions (19)(20)) may be written as follows:

$$
k_{\mathrm{Ps}, \mathrm{R}}(T, t)=4 \pi D_{\mathrm{Ps}, \mathrm{R}}(T) R_{\mathrm{Ps}, \mathrm{R}} \cdot\left[1+\frac{R_{\mathrm{Ps}, \mathrm{R}}}{\sqrt{\pi D_{\mathrm{Ps}, \mathrm{R}} t}}\right],
$$

where $R_{\mathrm{Ps}, \mathrm{R}}=R_{\mathrm{Ps}}+R$ is the reaction radius of the $\mathrm{R}+$ Ps chemical reaction, $R_{i}$ is approximately the geometric radius of $\mathrm{R}$ reactant, and $R_{\mathrm{Ps}} \approx R_{U}+1 / \varkappa$ is the radius of delocalization of the Ps wave function $(1 / \varkappa$ is the underbarrier penetration length of the Ps center-of-mass wave function; here, we consider Ps as a point particle in a potential well) [33].

The temperature dependence of the rate constant arises through the diffusion coefficients of the reagents, $D_{\mathrm{Ps}, \mathrm{R}}(T)=$ $D_{\mathrm{Ps}}+D_{\mathrm{R}}$. According to the Einstein relationship and the Stokes formula, the diffusion coefficients may be expressed as

$$
D_{\mathrm{R}}=\frac{T}{6 \pi \eta(T) R}, \quad D_{\mathrm{Ps}}=\frac{T}{4 \pi \eta(T) R_{\mathrm{Ps}}},
$$

where $\eta$ is the viscosity of the medium and $R$ and $R_{\mathrm{Ps}}$ are the hydrodynamic radii of $\dot{R}$ particle and Ps bubble. Although the applicability of these expressions on the atomic scale might be questionable, our studies in neat water $[18,33]$ have shown that it remains valid within a reasonable accuracy.

The knowledge of the surface tension of the liquid together with the Ps bubble model allows one to estimate the equilibrium radius of the Ps bubble and calculate the pick-off annihilation rate $\lambda_{\mathrm{po}}(t, T)$, which is of utmost importance to interpret the PAL spectra:

$$
\lambda_{\text {po }}(t, T)=\lambda_{p} \cdot P\left(R_{U}(t, T)\right) .
$$

Here, $\lambda_{p}$ is the "free" positron annihilation rate and $P\left(R_{U}\right)$ is the under-barrier penetration probability of $e^{+}$into a bulk of the liquid (into space containing molecular electrons). Its calculation is discussed below.

4.2. Low-Temperature Region. In this region, the Ps bubble formation time is larger than the $e^{+}$and Ps annihilation lifetimes. On a scale of $1 \mathrm{~ns}$, the size of a preexisting void in which a quasifree Ps has been localized does not change, since $t_{\text {bubble }} \gg 1 \mathrm{~ns}$. This situation is similar to that existing in polymers. Typically at these $T$ the diffusion time $t_{\text {diff }}$ is also much larger than the o-Ps lifetime ( 1ns), so one may completely neglect the diffusion motion of radiolytic products. For example in glycerol it happens at $T<30^{\circ} \mathrm{C}$, Figure 9.

To describe correctly the PAL spectra, one should average the pick-off annihilation kinetics over the size distribution of the preexisting voids in which Ps localization takes place. This can be done by using the theory of free volume entropy fluctuations [34], which claims that every molecule possesses some free volume $v_{i}$ with the probability

$$
\propto \exp \left(-\frac{v_{i}}{v_{F}}\right), \quad v_{F}(T) \approx v_{\mathrm{WS}}(T)-v_{\mathrm{vdW}},
$$

where $v_{F}$ is a difference between the volume of the WignerSeitz cell and van-der-Waals volume of a molecule. Because of the approximate character of this expression, $v_{F}$ may be considered as an adjustable parameter derivable by fitting the spectra in the low-temperature region. Note that in some cases the free volume distribution in the studied substances 
may be more complicated (nanocavities may have some selected sizes related to a particular chemical structure and bonding) [35].

If $v$ is the volume of a preexisting void in which the Ps atom can localize, the probability to find such a void is proportional to $\exp \left(-v / v_{F}\right)$. Neglecting the influence of any intratrack reactions (because of low $T$ and not discussing their possible tunneling nature), the calculation of the Ps pick-off annihilation kinetics reduces to averaging the exponents that relate to the pick-off annihilation of individual Ps atoms located at different voids:

$$
\left\langle e^{-\lambda_{\mathrm{po}}\left(R_{U}\right) t}\right\rangle=\int_{v_{\min }}^{\infty} e^{-\lambda_{\mathrm{po}}\left(R_{U}\right) t} \cdot e^{-v / v_{F}} \cdot \frac{d v}{v_{F}}, \quad v=\frac{4 \pi R_{U}^{3}}{3} .
$$

Here, $v_{\min }$ is the volume of the minimal cavity, which may trap Ps (Section 4).

4.3. Intermediate Temperatures. The most complex task in the PAL data processing refers to the intermediate temperature region, where the bubble formation time is comparable with the $e^{+}$lifetime in the medium (fortunately this $T$ interval is rather narrow because of "exponential" $T$-dependence of viscosity). qf-Ps localizes in one of the preexisting voids, which further somewhat increases in size; the time at which the equilibrium volume is reached depends on $T$. Obviously, the quantum-mechanical pressure exerted by Ps on the wall and thus the growth of the void cease when $e^{+}$annihilates.

At these temperatures, the Ps fate may also depend on the intratrack reactions. Therefore, the corresponding chemical kinetic equations must be solved by assuming that Ps initially localizes in a preexisting void of a given radius, which will increase with time until annihilation; the process must be averaged over the size distribution of the preexisting voids [32].

4.4. Interpretation of the PAL Spectra in Liquid Water at Different $T$. To illustrate briefly how the blob model works, we mention the solution of the problem with a puzzling temperature behavior of the long-lived component in water, Figure 8 . We have rejected conventional exponential deconvolution of the spectra and explicitly took into account the intrablob processes mentioned above [13, 18]. The decrease of $\tau_{3}$ versus $T$ was ascribed to the increasing efficiency of the oxidation (19) and ortho-paraconversion (20) reactions between Ps and intratrack radicals (mainly $\mathrm{OH}$ radicals). All parameters included in the model have a clear physical meaning: the Ps oxidation reaction radius $R_{\mathrm{Ox}} \approx R_{\mathrm{Ps}}+R_{\mathrm{OH}}$ which enters the rate constant of the Ps oxidation reaction (19), relative contact density $\eta_{c}$ in the Ps bubble state, free positron annihilation rate, the Ps formation rate constant, and some others (5 adjustable parameters in total).

It is seen from Figure 10 that the blob model explains well the experimental data in a wide range of temperatures (and magnetic fields also [13]). It does not lead to contradictions with known radiation chemistry data. The agreement between theory and experiment became even better when taking into account the time dependence of the Ps reaction rate coefficients. The temperature dependence of the Ps diffusion-controlled reaction rate constants agrees with the Stokes-Einstein law. Good fitting of the PAL data is obtained when the Ps hydrodynamic radius is equal to the radius $R_{\mathrm{Ps}}=R_{U}+1 / \varkappa$ of the Ps bubble (here $1 / \varkappa \approx$ $0.5 \AA$ is the under-barrier penetration depth of the Ps wave function).

\section{Ps Bubble Models}

Typical lifetimes of a parapositronium atom in condensed medium are about $130-180$ ps. They are close to the pPs lifetime in vacuum ( $125 \mathrm{ps})$. The orthopositronium lifetime in a medium is considerably shorter (about 100 times; some ns) in comparison with that in vacuum. This is due to the so-called pick-off process-prompt $2 \gamma$-annihilation of the $e^{+}$, composing Ps atom, with one of the nearest $e^{-}$of surrounding molecules, whose spin is antiparallel to the $e^{+}$spin. Just this property turns Ps into a nanoscale structural probe of matter. The theoretical task consists in calculating the pick-off annihilation rate $\lambda_{\text {po }}$, that is, in relating $\lambda_{\text {po }}$ with such properties of the medium like surface tension, viscosity, external pressure, and size of the Ps trap.

Originally, to explain the unexpectedly long lifetime of the ortho-Ps atom in liquid helium, Ferrel [36] suggested that due to the quantum mechanical nature of the Ps atom, it forms a nanobubble around itself. This is caused by a strong exchange repulsion between the o-Ps electron and electrons of the surrounding He atoms. Ferrel approximated this repulsion by a spherically symmetric potential barrier of radius $R_{\infty}$. To estimate the equilibrium radius of the Ps bubble he minimized the sum of the Ps energy in a spherically symmetric potential well, that is, $E_{\mathrm{Ps}}=\pi^{2} \hbar^{2} / 4 m R_{\infty}^{2}=$ $(\mathrm{Ry} / 2)\left(\pi a_{B} / R_{\infty}\right)^{2}$, Ry $=13.6 \mathrm{eV}$, and the surface energy, $4 \pi R_{\infty}^{2} \sigma$, where $\sigma$ is the macroscopic surface tension coefficient. The following relationship is hereby obtained for the equilibrium radius of the bubble:

$$
\frac{\mathrm{Ry}}{2} \cdot \frac{\pi^{2} a_{B}^{2}}{R_{\infty}^{2}}+4 \pi R_{\infty}^{2} \sigma \longleftrightarrow \min \text { over } R_{\infty} \Longrightarrow R_{\infty}=a_{B}\left(\frac{\pi \mathrm{Ry}}{8 \sigma a_{B}^{2}}\right)^{1 / 4} .
$$

5.1. The Tao-Eldrup Model. Ferrel's idea got further development in the studies of Tao [37] and Eldrup et al. [38]. They considered the Ps atom as a point particle in a liquid, that is, in a structureless continuum, Figure 11. The repulsive Ps-liquid interaction was approximated by a rectangular infinitely deep spherically symmetric potential well of radius $R_{\infty}$. In such a well, the wave function of a point particle has the following standard expression:

$$
\Psi\left(0 \leq r \leq R_{\infty}\right)=\frac{\sin \left(\pi r / R_{\infty}\right)}{\sqrt{2 \pi R_{\infty}} r}, \quad \Psi\left(r \geq R_{\infty}\right)=0 .
$$

Here, $r$ is the Ps center-of-mass coordinate. Because the Ps wave function equals zero at the bubble radius (and 


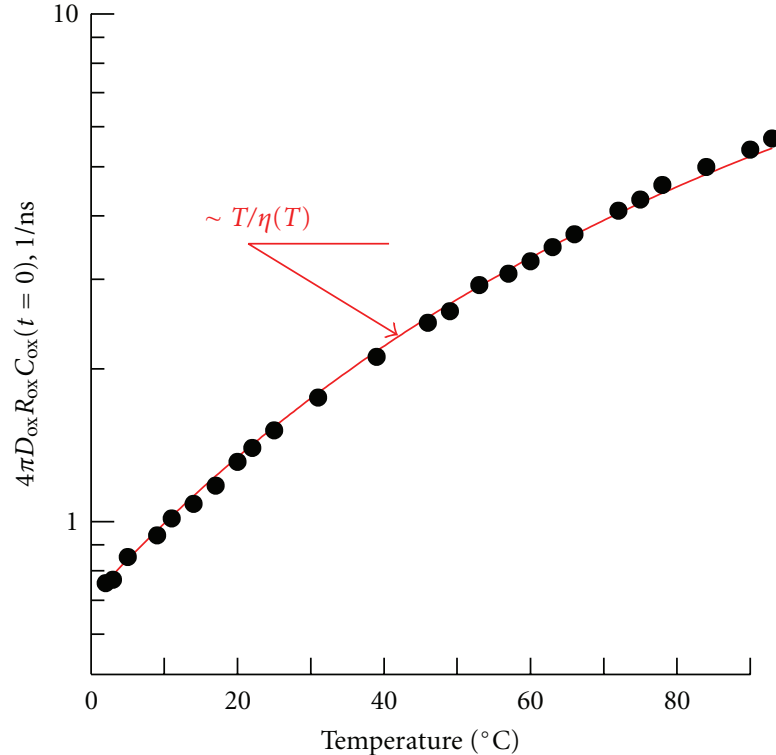

(a)

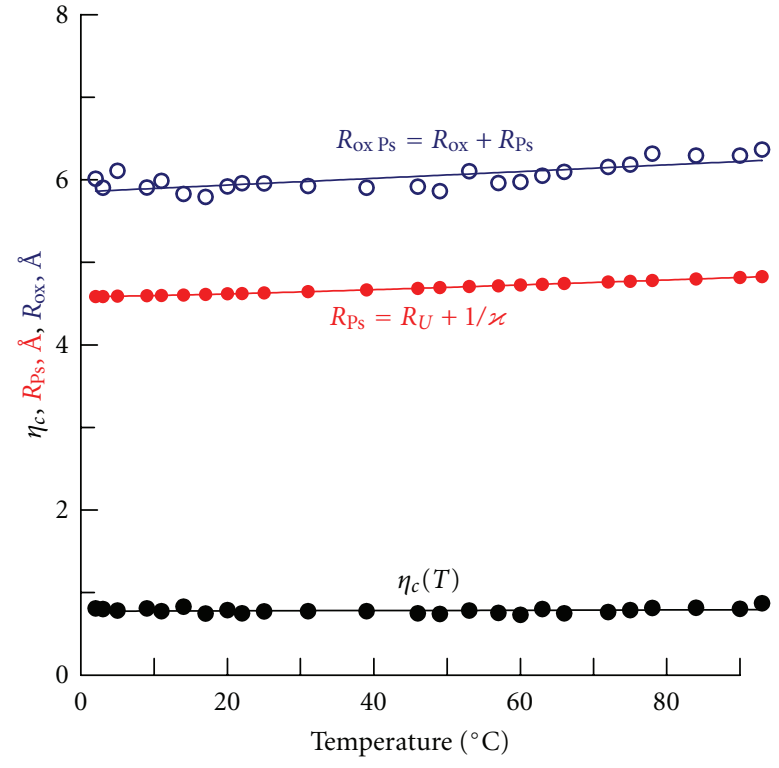

(b)

Figure 10: (a) Temperature dependence of the Ps oxidation reaction rate constant (at an infinite time, $t \rightarrow \infty$ ) multiplied by the "initial" concentration of oxidizers $c_{\mathrm{ox}}(0)$ in the $e^{+}$blob $\left(\mathrm{OH}\right.$ and $\mathrm{H}_{3} \mathrm{O}^{+}$, see $[13,18]$ for details). $D_{\mathrm{ox}}$ is the sum of $D_{\mathrm{Ps}}$ and the diffusion coefficient of the oxidizer ( $\mathrm{OH}$ radical, $\left.D_{\mathrm{OH}} \approx 1.5 \cdot 10^{-5} \mathrm{~cm}^{2} / \mathrm{s}\right)$. The obtained $T$-dependence agrees well with the Stokes-Einstein law, $\propto T / \eta(T)$. $(\mathrm{b})$ $T$-dependences of the oxidation reaction radius, $R_{\mathrm{ox}, \mathrm{Ps}}=R_{\mathrm{ox}}+R_{\mathrm{Ps}}$, the Ps radius, $R_{\mathrm{Ps}_{\mathrm{s}}}=R_{U}+1 / \kappa$, and the relative contact density parameter $\eta_{c} \approx 0.8$ in Ps. $R_{\mathrm{ox}}$ is the radius of the oxidizer (approximately, the geometric radius of the $\mathrm{OH}$ radical, $1.4 \AA$ ).

outside), there is no $e^{+}$overlapping with outer electrons of a medium. So, pick-off annihilation is absent. To overcome this difficulty it was postulated that molecular electrons, which form a "wall" of the Ps bubble, may penetrate inside the potential well. This results in the appearance of a surface layer of thickness $\delta=R_{\infty}-R$ having the same average electron density as in the bulk. As a result, the pick-off annihilation rate $\lambda_{\text {po }}$ becomes nonzero. It is proportional to the $e^{+}$overlapping integral with the electrons inside the bubble:

$$
\begin{aligned}
\lambda_{\mathrm{po}}=\lambda_{+} P_{R}, \quad P_{R} & =\int_{R}^{R_{\infty}}|\Psi(r)|^{2} 4 \pi r^{2} d r \\
& =\frac{\delta}{R_{\infty}}-\frac{\sin \left(2 \pi \delta / R_{\infty}\right)}{2 \pi} .
\end{aligned}
$$

This is the well-known Tao-Eldrup formula. Here, $\lambda_{+} \approx$ $2 \mathrm{~ns}^{-1}$ is the $e^{+}$annihilation rate in an unperturbed medium (it is proportional to Dirac's $2 \gamma$-annihilation cross-section and the number density of valence electrons). The thickness $\delta$ of the electron layer is an empirical parameter, which may have different values in various media.

Substituting (27) for $R_{\infty}$ into (29), one obtains the relationship between $\lambda_{\text {po }}$ and $\sigma$ with one adjustable parameter, $\delta$. It may be easily obtained by fitting experimental pick-off annihilation rate constants with the relationship (29), Figure 12. Thus, we obtain $\delta \approx 1.66 \AA$. Equation (29) with this value of $\delta$ is widely used for recalculation of the observed pick-off annihilation rate into the free volume $4 \pi R^{3} / 3$ of the cavity, where Ps atom resides and annihilates.

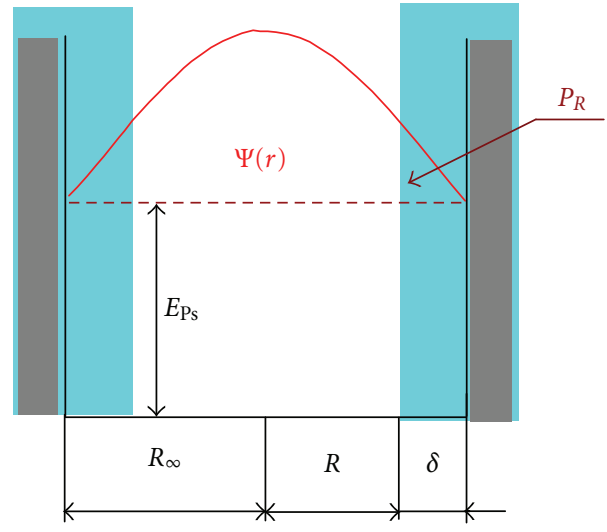

Figure 11: Tao-Eldrup model of Ps atom in a liquid phase. It is assumed that Ps is confined in an infinite spherically symmetric potential well of radius $R_{\infty} . \Psi(r)$ is the center-of-mass wave function of Ps. $R$ is the free volume radius of the Ps bubble. $\delta$ is the penetration depth of the molecular electrons into the Ps bubble.

\subsection{Further Development of the Ps Bubble Models: "Nonpoint"} Positronium. Along with the development of the "infinite potential well" Ps bubble model, another approach based on the finite potential well approximation was also elaborated [39-43]. However in both approaches, the Ps atom was approximated by a point particle. This leads to a significant simplification, but it is not justified from a physical viewpoint, because (1) the size of the localized state of Ps (size of the Ps bubble) does not significantly exceed the 


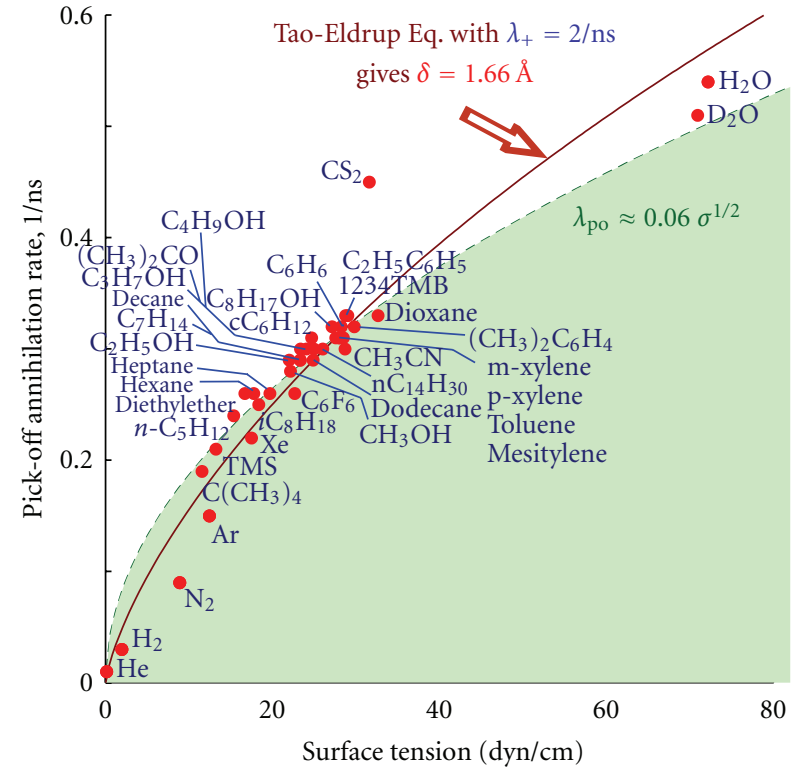

Figure 12: Dependence of the experimental pick-off annihilation rate constants [39] versus surface tension in different liquids. The solid curve shows the correlation given by the Tao-Eldrup at $\lambda_{+}=$ $2 \mathrm{~ns}^{-1}$ and optimal value $\delta=1.66 \AA$ (obtained from fitting of these data by means of (29)). The dashed curve illustrates the simplest approximation $\lambda_{\text {po }} \propto \sigma^{1 / 2}$.

distance between $e^{+}$and $e^{-}$in Ps (2) during the formation of the Ps bubble there is a substantial variation of the Ps internal energy (particularly of the Coulombic attraction of $e^{+}$and $e^{-}$), which is completely ignored in the "point-like" Ps models. In a vacuum or in a large bubble, the internal energy of Ps tends to $-\mathrm{Ry} / 2=-6.8 \mathrm{eV}$. In a continuous liquid (no bubble) with the high-frequency dielectric permittivity $\varepsilon \approx n^{2}(n \approx 2-3$ is the refractive index $)$ the energy of the Coulombic attraction between $e^{+}$and $e^{-}$decreases in absolute value by a factor $\varepsilon^{2} \approx 4-9$. The same takes place with the total Ps binding energy, which tends to the value $-\mathrm{Ry} / 2 \varepsilon^{2} \approx-(0.8-1.7) \mathrm{eV}$ (this is a simple consequence of the scaling $e^{2} \rightarrow e^{2} / \varepsilon$ of the Schrödinger equation for Ps atom). Thus, the change in the Ps internal energy during Ps formation may reach $5 \mathrm{eV}$. Obviously, this represents an important contribution to the energetics of Ps formation. Now we shall present a more accurate estimation of this contribution, which has not been done yet.

There is only a small number of papers where the consequences of the finite size of Ps are discussed in application to positron annihilation spectroscopy. To calculate $\lambda_{\text {po }}$, the Kolkata group [44, 45] suggested to smear the Ps atom over the relative $e^{+}-e^{-}$coordinate exactly in the same way as it is in a vacuum. Such an approach is valid for rather large bubbles. However, they do not discuss the variation of the internal Ps energy.

In [46-48] a path integral Monte Carlo technique was used to simulate the two-particle $e^{+}-e^{-}$system. However, to proceed with calculations the authors need potentials describing $e^{-}$-atom and $e^{+}$-atom interactions (they were taken from a variety of sources). However, the question about

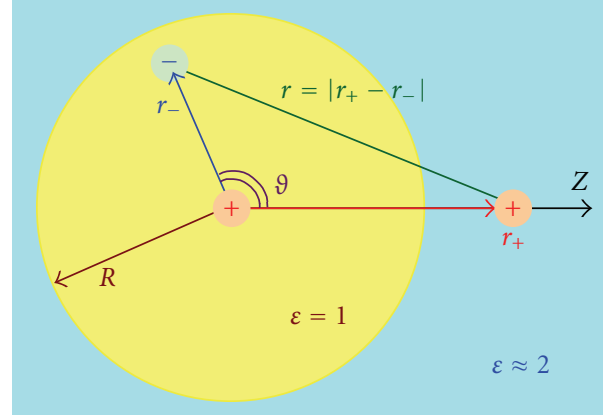

Figure 13: Coordinates of the $e^{+} e^{-}$pair.

modification of the $e^{+}-e^{-}$interaction because of the presence of the medium remains open (in this paper we roughly take this effect into account by means of introducing the high frequency dielectric permittivity).

In [49] the Ps atom is considered as a finite sized $e^{-} e^{+}$ pair, but the variation of the Coulombic interaction because of dielectric screening is not discussed. It was assumed that $e^{-}$is confined in an infinite potential well and $e^{+}$is bound to it by means of the Coulombic attraction. The wave function of the pair was taken as a series of orthogonal polynomials, their weights being determined from a minimization procedure of the total energy of the pair.

5.3. Hamiltonian of the $e^{+} e^{-}$Pair in a Medium. Let the $e^{+} e^{-}$ pair (Ps atom) have already formed in a liquid a nanobubble (spherical cavity; Ps bubble) of radius $R$ (the onset of coordinates is taken at the center of the bubble, Figure 13). Together with the molecules surrounding the $e^{+} e^{-}$pair, one has to deal with a quite intricate many-body problem with a complex hamiltonian. We reduce it to the following form:

$$
H \approx-\frac{\hbar^{2}\left(\Delta_{+}+\Delta_{-}\right)}{2 m_{e}}+U\left(r_{+}\right)+U\left(r_{-}\right)-U_{c}\left(r_{+}, r_{-}, R, \varepsilon\right) .
$$

Terms with Laplacians $\Delta_{+}$and $\Delta_{-}$over $r_{+}$and $r_{-}\left(e^{+}\right.$and $e^{-}$ coordinates) stand for the kinetic energies of the particles. $U\left(r_{+}\right)$and $U\left(r_{-}\right)$describe the individual interaction of $e^{+}$ and $e^{-}$with the medium. For them we adopt the following approximation:

$$
U\left(r_{+}\right)=\left\{\begin{array}{ll}
0, & r_{+}<R, \\
V_{0}^{+}, & r_{+}>R,
\end{array} \quad U\left(r_{-}\right)= \begin{cases}0, & r_{-}<R, \\
V_{0}^{-}, & r_{-}>R .\end{cases}\right.
$$

Here, $V_{0}^{+}$and $V_{0}^{-}$are the $e^{+}$and $e^{-}$work functions, respectively, Table 2 . The work function is introduced as the energy needed for an excess particle to enter the liquid without any rearrangement of its molecules and to stay there in a delocalized state, having no preferential location in a bulk. One may say that $V_{0}^{+}$and $V_{0}^{-}$are the ground state energies of the quasifree $e^{+}$and $e^{-}$, because their energies at rest after having been removed from the liquid to infinity are defined to be zero. 
Experimental values for $V_{0}^{-}$are known for many liquids (Table 2). Because of a lack of experimental data on the $e^{+}$ work functions, we admit that $V_{0}^{+} \approx V_{0}^{-}$and $\left|V_{0}^{+}+V_{0}^{-}\right| \approx 0$. Note that the variation of the internal energy of the pair, $\approx \operatorname{Ry}\left(1-1 / \varepsilon^{2}\right) / 2 \approx 5 \mathrm{eV}$, related with the variation in the dielectric screening of the $e^{+}-e^{-}$attraction in the Ps bubble formation process, very probably significantly exceeds $V_{0}^{-}$ and $V_{0}^{+}$.

In (30) $U_{c}$ stands for the Coulombic interaction between $e^{+}$and $e^{-}$in a polarizable medium. Assuming that the medium has the high frequency dielectric permittivity $\varepsilon$ of the bulk and a spherical cavity of radius $R$ (inside the cavity $\varepsilon=1$ ), one may calculate $U_{c}$ by solving the Poisson equation. Denoting the $e^{+}$and $e^{-}$coordinates as $r_{+}$and $r_{-}, U_{c}$ may be written in the form of the following series via the Legendre polynomials $P_{l}(x=\cos \theta)$ [50]:

$$
\begin{aligned}
& \frac{U_{c}\left(r_{+}<R, r_{-}<R\right)}{\mathrm{Ry}}=\frac{2 a_{B}}{r}-\left(1-\frac{1}{\varepsilon}\right) \frac{2 a_{B}}{R} \\
& \times\left(1+\sum_{l=1}^{\infty} \frac{(1+l) P_{l}(x)}{1+l+l / \varepsilon} \cdot \frac{r_{+}^{l} r_{-}^{l}}{R^{2 l}}\right), \\
& \frac{U_{c}\left(r_{+}<R, r_{-}>R\right)}{\mathrm{Ry}}=\frac{2 a_{B}}{\varepsilon r_{-}}\left(1+\sum_{l=1}^{\infty} \frac{(1+2 l) P_{l}(x)}{1+l+l / \varepsilon} \cdot \frac{r_{+}^{l}}{r_{-}^{l}}\right), \\
& \frac{U_{c}\left(r_{+}>R, r_{-}>R\right)}{\mathrm{Ry}}=\frac{2 a_{B}}{\varepsilon r}+\left(1-\frac{1}{\varepsilon}\right) \frac{2 a_{B}}{R} \sum_{l=1}^{\infty} \frac{l P_{l}(x)}{l+\varepsilon+l \varepsilon} \\
& \frac{\cdot\left(\frac{R^{2}}{r_{+} r_{-}}\right)^{I+1} ;}{\mathrm{Ry}}=\frac{2 a_{B}}{\varepsilon r_{+}}\left(1+\sum_{l=1}^{\infty} \frac{(1+2 l) P_{l}(x)}{1+l+l / \varepsilon} \cdot \frac{r_{-}^{l}}{r_{+}^{l}}\right) .
\end{aligned}
$$

Here, the argument of the Legendre polynomials is $x \equiv \cos \vartheta$, where $\vartheta$ is the angle between the $z$ axis and the direction of $r_{-}$.

5.4. Wave Function of the $e^{+} e^{-}$Pair and Minimization of Its Total Energy $\langle H\rangle$. Keeping in mind further use of the variational procedure, let us choose the normalized $e^{+} e^{-}$ wave function in the following simplest form:

$$
\begin{gathered}
\Psi_{+-}\left(r_{+}, r_{-}\right)=\frac{\exp \left(-r / 2 a-r_{\mathrm{cm}} / 2 b\right)}{8 \pi \sqrt{a^{3} b^{3}}}, \\
\mathbf{r}_{\mathrm{cm}}=\frac{\mathbf{r}_{+}+\mathbf{r}_{-}}{2}, \quad \mathbf{r}=\mathbf{r}_{+}-\mathbf{r}_{-} .
\end{gathered}
$$

In both cases of a rather large bubble and a uniform dielectric continuum, $\Psi_{+-}$breaks into a product of two terms: the first one depends on the distance $r$ between $e^{+}$and $e^{-}$, and the second one depends on the center-of-mass coordinate $\mathbf{r}_{\mathrm{cm}}$. Parameters $a$ and $b$ are the variational ones, over which we have minimized the energy of the $e^{+} e^{-}$pair:

$$
E(a, b, R)=\left\langle\Psi_{+-}|H| \Psi_{+-}\right\rangle \longrightarrow \min \Longrightarrow a(R), b(R) .
$$

The simplest verification of the calculations is to recover two limiting cases. In case of large bubbles $(R \rightarrow \infty)$, one should reproduce the "vacuum" state of the Ps atom: its total energy must tend to $-\mathrm{Ry} / 2=-6.8 \mathrm{eV}$, the kinetic energy to $+\mathrm{Ry} / 2$, and the Coulombic energy to - Ry. In case of small bubbles $(R \rightarrow 0)$, the delocalized qf-Ps state must be reproduced. The Schrödinger equation for qf-Ps has the same form as for the vacuum Ps, but with the substitution $e^{2} \rightarrow e^{2} / \varepsilon$. Then the total qf-Ps energy tends to $V_{0}^{+}+V_{0}^{-}-\mathrm{Ry} / 2 \varepsilon^{2}$, its kinetic part tends to $+\mathrm{Ry} / 2 \varepsilon^{2}=1.7 \mathrm{eV}(\varepsilon=2)$, and the Coulombic energy tends to $-\mathrm{Ry} / \mathcal{\varepsilon}^{2}=-3.4 \mathrm{eV}$. Figure 14 displays optimal values of $a$ and $b$ as well as different contributions to the total energy of the $e^{+} e^{-}$pair.

5.5. Relative Contact Density and Pick-Off Annihilation Rate. Using the wave function (33) it is easy to obtain the relative contact density $\eta_{c}$ in the Ps atom:

$$
\eta_{c}=\frac{\iint d^{3} r_{+} d^{3} r_{-}\left|\Psi_{+-}\left(r_{+}, r_{-}\right)\right|^{2} \delta\left(r_{+}-r_{-}\right)}{\iint d^{3} r_{+} d^{3} r_{-}\left|\Psi_{+-}^{\mathrm{vac}}\left(r_{+}, r_{-}\right)\right|^{2} \delta\left(r_{+}-r_{-}\right)}=\frac{a_{B}^{3}}{a^{3}(R)} .
$$

This quantity determines the observable Ps annihilation rate constant (including the case with applied permanent magnetic field). The resulting dependencies of $\eta_{c}$ are shown in Figure 14 (on the left). Because, for qf-Ps, parameter $a$ is equal to $\varepsilon a_{B}$, for qf-Ps the value of $\eta_{c}$ should be $1 / \varepsilon^{3}=1 / 8$, which is well recovered in numerical calculations. When $R$ increases, $\eta_{c}$ approaches unity, because $a$ tends to its vacuum value $a_{B}$.

Knowing the expression for the wave function (33), one may calculate the positron overlapping $P_{R}$ with molecular electrons, surrounding the Ps atom, and therefore find out the pick-off annihilation rate constant:

$$
\lambda_{\mathrm{po}}(R) \approx \lambda_{+} P_{R}, \quad P_{R} \approx \int_{r_{+}>R} d^{3} r_{+} \int d^{3} r_{-}\left|\Psi_{+-}\left(r_{+}, r_{-}\right)\right|^{2} .
$$

Here, $\lambda_{+} \approx 2 \mathrm{~ns}^{-1}$ is the annihilation rate constant of "free" positrons. Results of calculations of $\lambda_{\text {po }}(R)$ for optimal $a$ and $b$ values, which correspond to the minimal Ps energy at a given $R$, are shown in Figure 15.

5.6. Discussion on the Nonpoint Ps Approximation. It is usually considered that $\mathrm{Ps}$ is a solvophobic particle, that is, it forms a bubble when entering a liquid because of exchange repulsion between $e^{-}$in Ps and the surrounding molecular electrons. If both $V_{0}^{+}$and $V_{0}^{-}$are negative, that is, $e^{+}$and $e^{-}$consider a cavity as a potential barrier, they are pulled to the bulk by polarization interaction with the medium. Nevertheless, even in this case the Ps bubble may be formed due to an enhancement of the Coulombic $e^{+} e^{-}$attraction inside the cavity (no dielectric screening inside). This feature cannot be taken into account when Ps is simulated as a point particle.

It is seen that the behavior of the total energy of the pair (red curve in Figure 14) strongly differs from the Tao-Eldrup prediction (green dashed curve; the first term in (27), where $R_{\infty}$ is replaced by $R$ ), as well as from the expectation based on the finite potential well model (brown curve, Figure 14; 


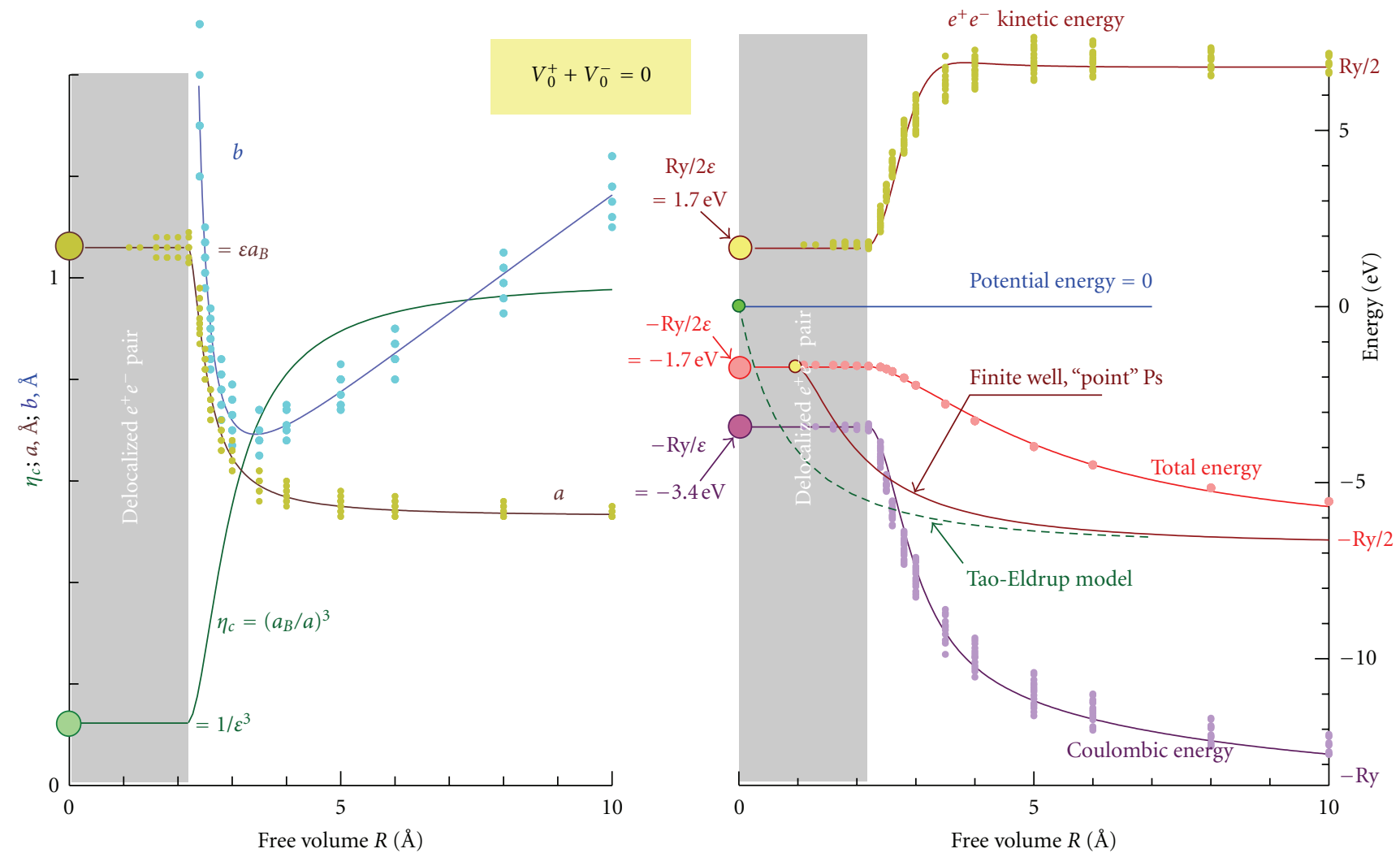

Figure 14: Dependences of the optimal parameters $a$ and $b$ versus $R$, the bubble radius. They enter the $e^{+} e^{-}$wave function and yield the minimum of the total energy $\langle H\rangle$. The relative contact density $\eta_{c}$ and different energy contributions to $\langle H\rangle$ (at optimal $a$ and $b$ ) are shown as well. It was assumed that $\varepsilon=2$.

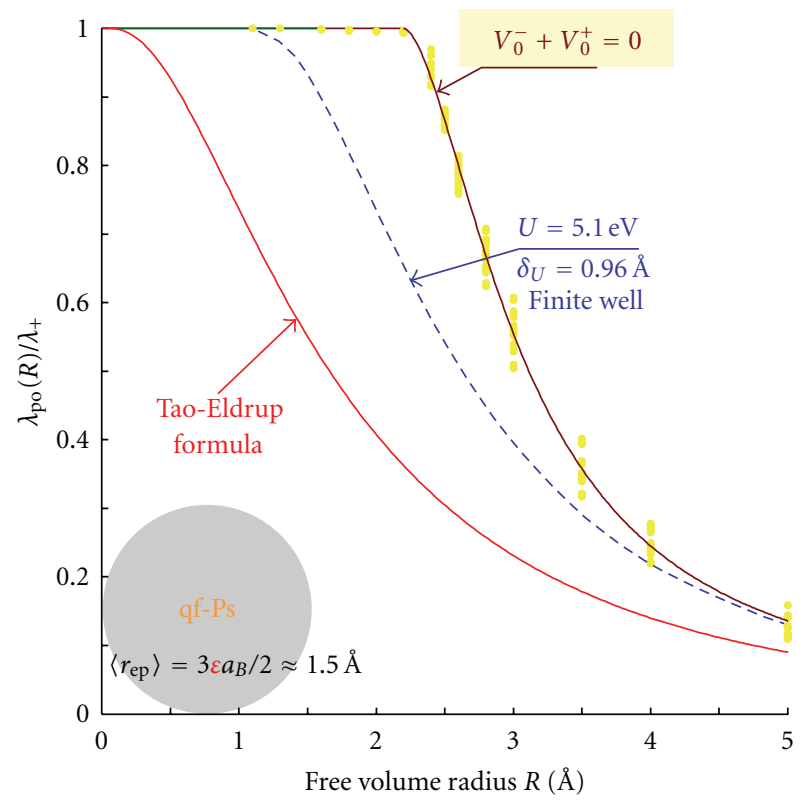

FIGURE 15: Pick-off annihilation rate constant of Ps, localized in a bubble of $R$ when $V_{0}^{+}+V_{0}^{-}=0$ (brown curve). For small $R(\lesssim 2.3 \AA$ ) the calculated values of $\lambda_{\text {po }}$ are equal to $\lambda_{+}$. The red line shows the pick-off annihilation rate constant, calculated according to the Tao-Eldrup formula. The dashed line is the calculation according to the finite potential well model (for comparison we adopted that the depth of the well is $\left(1-1 / \mathcal{E}^{2}\right) \mathrm{Ry} / 2 \approx 5.1 \mathrm{eV}$ and its radius is $R$; in this case the minimal radius of the well when there appears an energy level for Ps is $\left.\delta_{U}=0.96 \AA\right)$. 
the Coulombic potential cannot be approximated well by a rectangular spherically symmetric potential). The same is true for the pick-off annihilation rate, Figure 15.

Calculations demonstrate one common feature: up to $R \lesssim 2.3 \AA$ all dependencies remain the same as in a medium without any cavity, but at larger $R$ there are significant deviations. This is related to the known quantum mechanical phenomenon-absence of a bound state of a particle in a small finite 3d-potential well. In such cavities, Ps cannot be bound, it does not exert any repulsive pressure on their walls, and it does not stimulate their transformation towards the equilibrium Ps bubble. The possibility of finding a suitable preexisting cavity, sufficient at least for preliminary localization of qf-Ps, may be a limiting factor for the formation of the Ps bubble state.

One may find an equilibrium Ps bubble radius by minimizing the sum of the total $e^{+} e^{-}$energy $\langle H\rangle$ and the surface energy of the bubble. For water it turns out to be 5$5.2 \AA$, which is about $2 \AA$ larger than predicted by the TaoEldrup model. For such a large bubble, the relative contact density is $\eta_{c} \approx 0.9$, Figure 14 . It is somewhat higher than the experimental values $(0.65-0.75$ [13]). This discrepancy may indicate that $e^{+}$and $e^{-}$really interact with a medium in a different way, for example, it could be $V_{0}^{+}>V_{0}^{-}$. It means that the positron may be really trapped by a cavity, and $e^{-}$ will be bound to the trapped $e^{+}$by the Coulombic attraction. This scenario may be also considered in the framework of the present approach, but the expression for the trial wave function of this pair must be modified from the "symmetric" form (33) (with respect to the $e^{+}$and $e^{-}$coordinates) to the "asymmetric" one:

$$
\Psi_{+-}\left(r_{+}, r_{-}\right) \approx \frac{\exp \left(-\left|r_{+}-r_{-}\right| / 2 a-r_{+} / 2 b\right)}{8 \pi \sqrt{a^{3} b^{3}}},
$$

which explicitly takes into account the $e^{+}$localization owing to the factor $\exp \left(-r_{+} / 2 b\right)$.

Actually, any Ps bubble model reduces the original manybody (multiparticle) problem to a simpler one, that of one or two particles in an external field, which simulates the interaction with the medium. To calculate this field one usually has to rely on some macroscopic approaches, whose validity remains uncertain. For example, in our case we have somehow related the actual arrangement of molecules around the Ps bubble with the jump of dielectric permittivity outside the bubble.

\section{Conclusion}

We have made an attempt to trace the fate of a positron in molecular liquids, starting from its entering the medium till annihilation with an electron. Considering the ionization slowing down of the projectile positron, one may conclude that the $e^{+}$blob (terminal part of the $e^{+}$track) contains several tens of ion-electron pairs, which are confined in a spherical region with a radius of several nanometers. Ionelectron pairs in the blob are bound together because of their mutual Coulombic attraction. This is why outdiffusion of the blob species obeys ambipolar diffusion law.
Subionizing $e^{+}$can escape from the blob. It becomes thermalized either outside the blob or inside it. Energetic restrictions have been shown to leave only one possibility to form Ps atom. It is the combination of the thermalized positron with one of the intrablob electrons (the recombination mechanism or the intratrack mechanism).

Primary ion-electron pairs transform into secondary radiolytic products (typical concentration $\gtrsim 0.01 \mathrm{M}$ ), which are chemically active and thus can interact with Ps: oxidize it and/or stimulate its ortho-paraconversion. Because the number of ion-electron pairs is large and the diffusion displacement of the species on a nanosecond timescale is comparable with the size of the blob (at least at a high- $T$ diffusion region), it appears inescapable to describe these processes on the basis of inhomogeneous diffusion kinetic equations in terms of concentrations of the species, rate constants, diffusion coefficients, and so forth.

In the case of water it is clearly seen that taking into account interactions with intratrack products (Ps oxidation by $\mathrm{OH}$ radicals and $\mathrm{H}_{3} \mathrm{O}^{+}$) is important. Otherwise, it is not possible to explain the experimentally observed decrease of the ortho-Ps lifetime with temperature.

Calculating the pick-off annihilation rate constant is of the utmost importance. This is the main goal of the Ps bubble models. Up to now most of calculations deal with the "point-like" positronium, by using its center-of-mass wave function only. In the framework of this approximation it is not possible to take into account the variation of the internal (Coulombic) energy of the $e^{+}-e^{-}$pair during Ps bubble formation. However, we have shown that this energy contribution is not small and may play a decisive role.

Finally, we would like to draw attention to one of the possible applications of the positron spectroscopy: fast detection of potentially carcinogenic chemical compounds. These substances are considered as one of the major causes of human cancer. Modern technologies have led to new potential chemical carcinogens with which people may be in contact in everyday life. There is a need for a fast and cheap method of testing such compounds. Positron annihilation spectroscopy may provide such a method $[51,52]$.

\section{Acknowledgment}

This work is supported by the Russian Foundation of Basic Research (Grant 11-03-01066).

\section{References}

[1] J. Dryzek and P. Horodek, "The distribution of slowing-down times of positrons emitted from ${ }^{22} \mathrm{Na}$ and ${ }^{68} \mathrm{Ge} \backslash{ }^{68} \mathrm{Ga}$ isotopes into metals," Materials Science Forum, vol. 666, pp. 10-14, 2011.

[2] H. G. Paretzke, "Radiation track structure theory," in Kinetics of Nonhomogeneous Processes, G. R. Freeman, Ed., pp. 89-170, John Wiley \& Sons, New York, NY, USA, 1987.

[3] V. M. Byakov and S. V. Stepanov, "Common features in the formation of $\mathrm{Ps}, \mathrm{Mu}$, radioltic hydrogen and solvated electrons in aqueous solutions," Journal of Radioanalytical and Nuclear Chemistry, vol. 210, no. 2, pp. 371-405, 1996. 
[4] V. M. Byakov and F. G. Nichiporov, Intratrack Chemical Processes, Energoatomizdat, Moscow, Russia, 1985.

[5] S. V. Stepanov, "Energy losses of subexcitation charged particles in polar media," Radiation Physics and Chemistry, vol. 46, no. 1, pp. 29-37, 1995.

[6] S. V. Stepanov and V. M. Byakov, "Physical and radiation chemistry of the positron and positronium," in Principles and Applications of Positron and Positronium Chemistry, Y. C. Jean, P. E. Mallone, and D. M. Schrader, Eds., chapter 5, World Scientific Publications, 2003.

[7] S. V. Stepanov and V. M. Byakov, "Electric field effect on positronium formation in liquids," Journal of Chemical Physics, vol. 116, no. 14, pp. 6178-6195, 2002.

[8] S. V. Stepanov, V. M. Byakov, C. L. Wang, Y. Kobayashi, and K. Hirata, "Electric field effect on Ps formation: black blob model," Materials Science Forum, vol. 363-365, pp. 392-394, 2001.

[9] S. V. Stepanov, V. M. Byakov, and Y. Kobayashi, "Ps formation in molecular media: effect of the external electric field," Physical Review B, vol. 72, no. 5, Article ID 054205, 2005.

[10] S. V. Stepanov and V. M. Byakov, "Ps formation in molecular media: low temperature hydrocarbons," Physica Status Solidi (C) Current Topics in Solid State Physics, vol. 4, no. 10, pp. 3684-3689, 2007.

[11] F. Bockstahl, I. Billard, G. Duplâtre, and A. Bonnenfant, "Positronium formation and quenching in frozen and liquid solutions in octanol," Chemical Physics, vol. 236, no. 1-3, pp. 393-403, 1998.

[12] M. Muhieddine, E. Canot, and R. March, "Various approaches for solving problems in heat conduction with phase," International Research Journal of Finance, vol. 6, no. 1, pp. 1-20, 2009.

[13] S. V. Stepanov, D. S. Zvezhinski, G. Duplâtre, V. M. Byakov, Y. Y. Batskikh, and P. S. Stepanov, "Incorporation of the magnetic quenching effect into the blob model of Ps formation. Finite sized Ps in a potential well," Materials Science Forum, vol. 666, pp. 109-114, 2011.

[14] K. Kotera, T. Saito, and T. Yamanaka, "Measurement of positron lifetime to probe the mixed molecular states of liquid water," Physics Letters A, vol. 345, no. 1-3, pp. 184-190, 2005.

[15] G. Duplâtre, A. Haessler, and J. C. Abbé, "Interactions of positronium with $\mathrm{Co}^{2+}$ in water: mechanisms and temperature effects," Journal of Physical Chemistry, vol. 89, no. 9, pp. 1756-1760, 1985.

[16] J. C. Abbé, G. Duplâtre, A. Haessler, A. Marques Netto, and D. Pilo Veloso, "Temperature and (polar) solvent effects on positronium reactions with nitroxyl free radicals," Journal of Physical Chemistry, vol. 88, no. 10, pp. 2071-2076, 1984.

[17] J. Talamoni, J. C. Abbé, G. Duplâtre, and A. Haessler, “Temperature effects on positronium formation and inhibition: a contribution to the elucidation of early spur processes-III. Aqueous solutions," Radiation Physics and Chemistry, vol. 20, no. 4, pp. 275-280, 1982.

[18] S. V. Stepanov, G. Duplâtre, V. M. Byakov, V. S. Subrahmanyam, D. S. Zvezhinskii, and A. S. Mishagina, "Influence of temperature on intratrack processes and Ps formation and behaviour in liquid water," Materials Science Forum, vol. 607, pp. 213-217, 2009.

[19] C. Bonacina, G. Comini, A. Fasano, and M. Primicerio, "Numerical solution of phase-change problems," International Journal of Heat and Mass Transfer, vol. 16, no. 10, pp. 18251832, 1973.

[20] R. C. Wilhoit, J. Chao, and K. R. Hall, "Thermodynamic properties of key organic oxygen compounds in the carbon range $C_{1}$ to $C_{4}$. Part 1 . Properties of condensed phases,"
Journal of Physical and Chemical Reference Data, vol. 14, no. 1, pp. 1-175, 1985.

[21] C. Yaws, Thermophysical Properties of Chemicals and Hydrocarbons, William Andrew, New York, NY, USA, 2008.

[22] O. A. Korolyuk, "Thermal conductivity of molecular crystals of monoatomic alcohols: from methanol to butanol," Fizika Nizkikh Temperatur, vol. 37, no. 5, pp. 526-530, 2011.

[23] C. Yaws, Lange's Handbook of Chemistry, McGraw-Hill, 15th edition, 1999.

[24] D. R. Lide, Handbook of Chemistry and Physics, CRC Press LLC, 84th edition, 2004.

[25] O. E. Mogensen, Positron Annihilation in Chemistry, Springer, Berlin, Germany, 1995.

[26] T. Tabata, Y. Ito, and S. Tagawa, Handbook of Radiation Chemistry, CRC Press, Boca Raton, Fla, USA, 1991.

[27] Y. Ito, "Radiation chemistry: intraspur effects and positronium formation mechanism," in Positron and Positronium Chemistry, D. M. Schrader and Y. C. Jean, Eds., Studies in Physical and Theoretical Chemistry Series, pp. 120-158, Elsevier Scientific Publishers, 1988.

[28] O. E. Mogensen, "Spur reaction model of positronium formation," Journal of Chemical Physics, vol. 60, no. 3, pp. 9981004, 1974.

[29] V. M. Byakov, V. I. Goldanskii, and V. P. Shantarovich, "About the possible role of "dry" electrons in positronium formation in a liquid," Doklady Physical Chemistry, vol. 219, no. 3, pp. 1090-1093, 1974, Doklady Akademii Nauk, SSSR, V. 219(3), pp. 633-636, 1974.

[30] V. M. Byakov, "The nature of the precursors of radiolytic molecular hydrogen in water, and the mechanism of positronium formation in liquids," International Journal for Radiation Physics and Chemistry, vol. 8, no. 3, pp. 283-288, 1976.

[31] K. V. Mikhin, S. V. Stepanov, and V. M. Byakov, "Dynamics of positronium bubble growth in liquid media and the energy dissipation problem," High Energy Chemistry, vol. 39, no. 1, pp. 36-43, 2005.

[32] S. V. Stepanov, D. S. Zvezhinskii, G. Duplâtre, V. M. Byakov, and V. S. Subrahmanyam, "A novel approach to the interpretation of PAL spectra in glycerol," Materials Science Forum, vol. 607, pp. 260-262, 2009.

[33] S. V. Stepanov, V. M. Byakov, G. Duplâtre, D. S. Zvezhinskiy, and Y. V. Lomachuk, "Positronium formation in a liquid phase: influence of intratrack reactions and temperature," Physica Status Solidi (C) Current Topics in Solid State Physics, vol. 6, no. 11, pp. 2476-2481, 2009.

[34] A. I. Burshtein, Molecular Physics, Nauka, Novosibirsk, Russia, 1986.

[35] S. K. Sharma, A. Zaydouri, G. Roudaut, and G. Duplâtre, "Effect of water on glass transition in starch/sucrose matrices investigated through positron annihilation lifetime spectroscopy: a new approach," Physical Chemistry Chemical Physics, vol. 13, pp. 19338-19344, 2011.

[36] R. A. Ferrell, "Long lifetime of positronium in liquid helium," Physical Review, vol. 108, no. 2, pp. 167-168, 1957.

[37] S. J. Tao, "Positronium annihilation in molecular substances," Journal of Chemical Physics, vol. 56, no. 11, pp. 5499-5510, 1972.

[38] M. Eldrup, D. Lightbody, and J. N. Sherwood, "The temperature dependence of positron lifetimes in solid pivalic acid," Chemical Physics, vol. 63, no. 1-2, pp. 51-58, 1981.

[39] S. V. Stepanov and V. M. Byakov, "What do positrons say about the structure and properties of fluids?" Journal of Structural Chemistry, vol. 43, no. 6, pp. 949-971, 2002. 
[40] A. T. Stewart and C. V. Briscoe, "Positron Annihilation," in Proceedings of the Conference International Symposium on Positron Annihilation Studies of Fluids, A. T. Stewart and L. O. Roellig, Eds., p. 383, Wayne State UniversityAcademic Press, New York, NY, USA, 1959.

[41] A. P. Buchikhin, V. I. Gol'danskii, A. O. Tatur, and V. P. Shantarovich, "The positronium atom in organic liquids," Journal of Experimental and Theoretical Physics, vol. 33, no. 3, article 615, 1971, Zh. Eksp. Teor. Fiz., Vol. 60(3), p. 1136, 1971.

[42] L. O. Roellig, "Positron annihilation in liquids and condensed gases," in Proceedings of Wayne State University Conference on Positron AnnihilationAcademic Press, A. T. Stewart and L. O. Roellig, Eds., p. 127, New York, NY, USA, 1967.

[43] C. Dauwe, N. Balcaen, S. Van Petegem, and D. Segers, "Trapping of positronium in a size-dependent spherical square well potential," Radiation Physics and Chemistry, vol. 58, no. 56, pp. 681-685, 2000.

[44] D. Dutta, B. N. Ganguly, D. Gangopadhyay, T. Mukherjee, and B. Dutta-Roy, "Corrections to the prevalent bubble model of positronium annihilation in liquids," Physical Review B, vol. 65, no. 9, Article ID 094114, 2002.

[45] D. Dutta, B. N. Ganguly, D. Gangopadhyay, T. Mukherjee, and B. Dutta-Roy, "General trends of positronium pickoff annihilation in molecular substances," Journal of Physics Condensed Matter, vol. 14, no. 32, pp. 7539-7549, 2002.

[46] B. N. Miller and T. Reese, "Path integral simulation of positronium," Nuclear Instruments and Methods in Physics Research B, vol. 192, no. 1-2, pp. 176-179, 2002.

[47] L. Larrimore, R. N. McFarland, P. A. Sterne, and A. L. R. Bug, "Two-chain path integral model of positronium," Journal of Chemical Physics, vol. 113, no. 23, pp. 10642-10650, 2000.

[48] A. L. R. Bug, M. Muluneh, J. Waldman, and P. A. Sterne, "Positronium in solids: computer simulation of pick-off and self-annihilation," Materials Science Forum, vol. 445-446, pp. 375-379, 2004.

[49] T. Fülöp, Z. Farkas, A. Seeger, and J. Major, "On the inner structure of confined positronium," http://arxiv.org/abs/condmat/0304442v1.

[50] V. V. Batygin and I. N. Toptygin, "Problems on electrodynamics," Tech. Rep. 157, NIC RKhD, Moscow, Russia, 2002.

[51] V. M. Byakov, S. V. Stepanov, and O. P. Stepanova, "Positron method for diagnostics of carcinogens," Materials Science Forum, vol. 607, pp. 223-226, 2009.

[52] V. M. Byakov, S. V. Stepanov, and O. P. Stepanova, "PAL spectroscopy and testing for potential carcinogens," Physica Status Solidi (C) Current Topics in Solid State Physics, vol. 6, no. 11, pp. 2503-2506, 2009. 


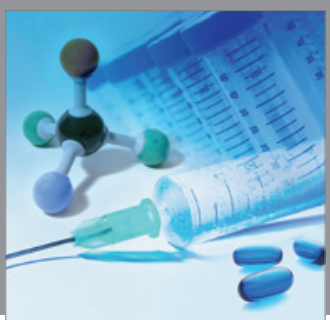

International Journal of

Medicinal Chemistry

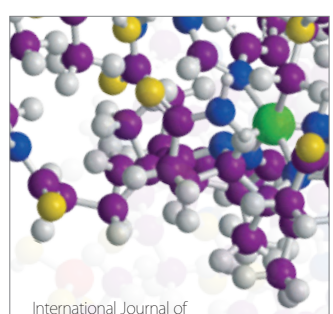

Carbohydrate Chemistry

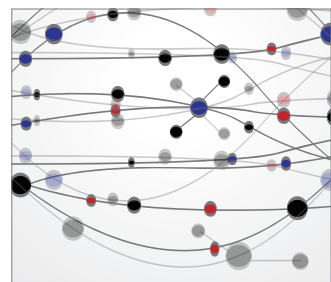

The Scientific World Journal
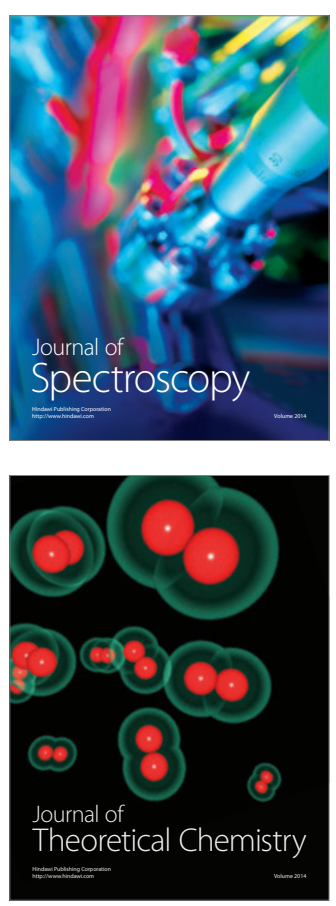
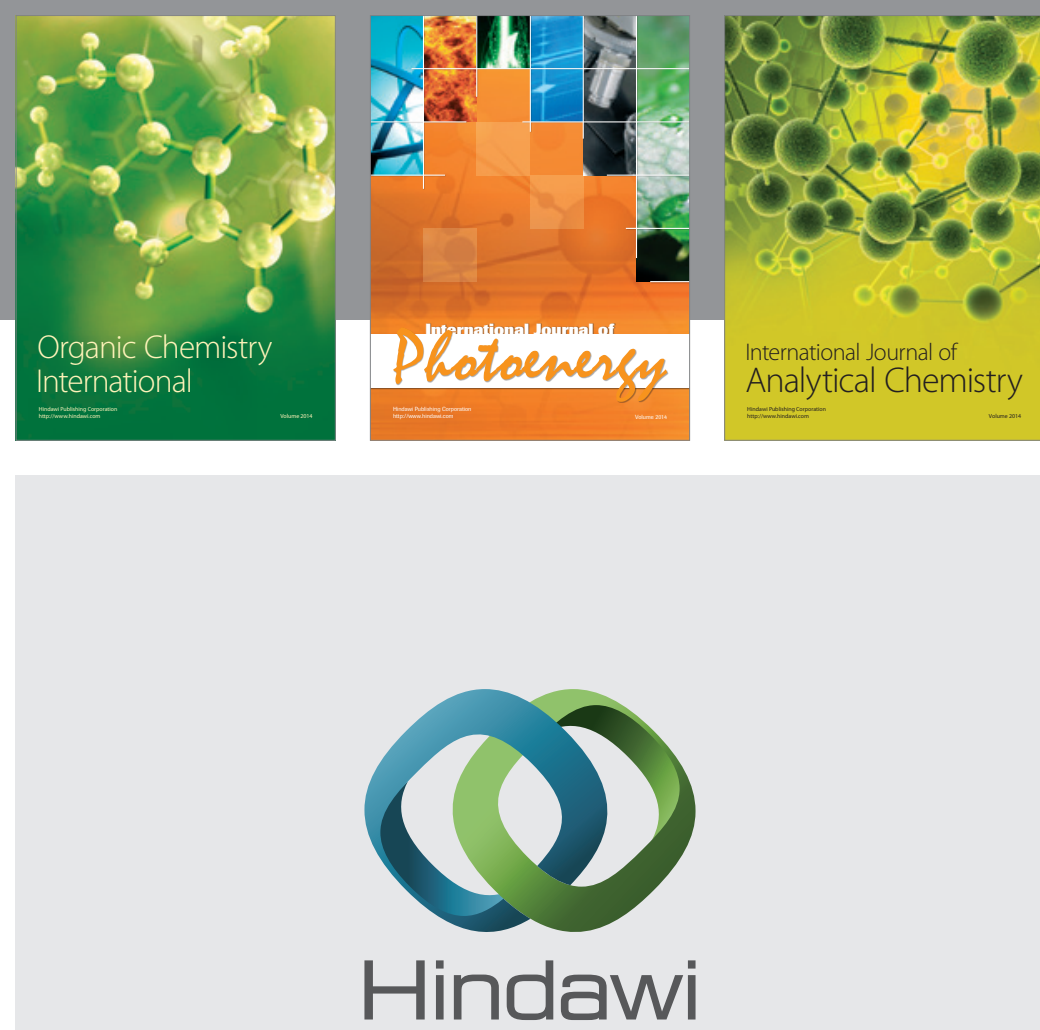

Submit your manuscripts at

http://www.hindawi.com
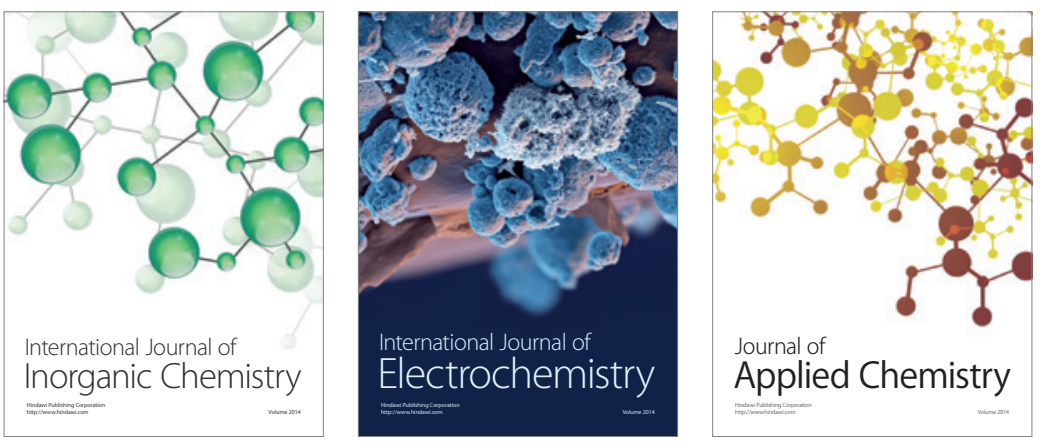

Journal of

Applied Chemistry
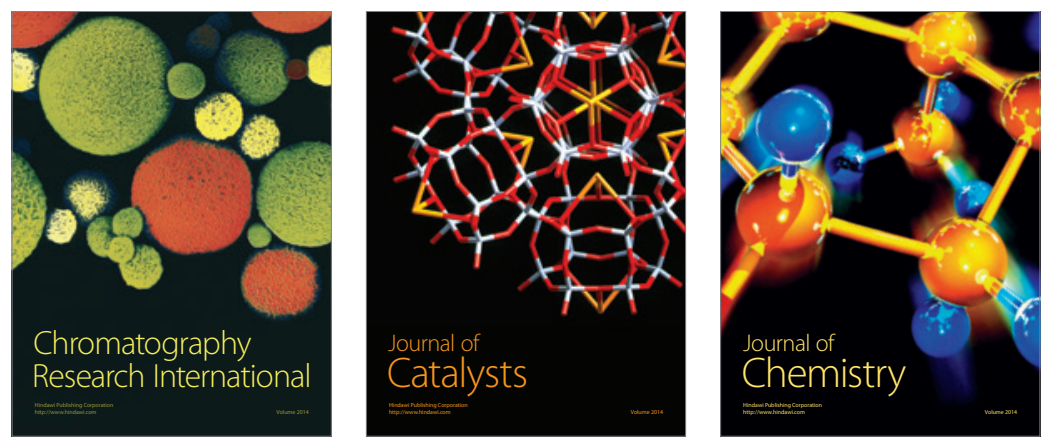
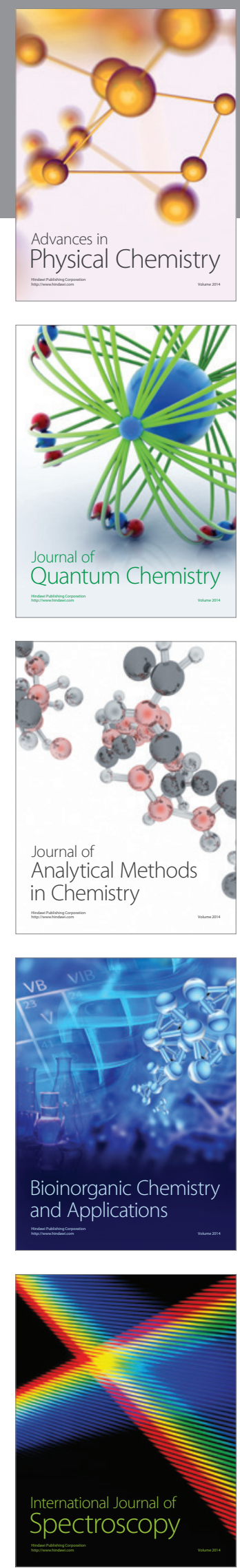